\title{
Region-specific H3K9me3 gain in aged somatic tissues in Caenorhabditis elegans
}

\author{
Cheng-Lin Li $\oplus^{1}$, Mintie Pu $\odot^{2}$, Wenke Wang ${ }^{1}$, Amaresh Chaturbedi ${ }^{1}$, Felicity \\ J. Emerson $\mathbb{D}^{1}$, Siu Sylvia Lee ${ }^{1 *}$ \\ 1 Department of Molecular Biology and Genetics, Cornell University, Ithaca, New York, United States of \\ America, 2 State Key Laboratory for Conservation and Utilization of Bio-Resources and Center for Life \\ Science, School of Life Sciences, Yunnan University, Kunming, Yunnan, China \\ * sylvia.lee@ cornell.edu
}

\section{G open ACcess}

Citation: Li C-L, Pu M, Wang W, Chaturbedi A, Emerson FJ, Lee SS (2021) Region-specific $\mathrm{H} 3 \mathrm{~K} 9 \mathrm{me} 3$ gain in aged somatic tissues in Caenorhabditis elegans. PLoS Genet 17(9): e1009432. https://doi.org/10.1371/journal. pgen.1009432

Editor: Anne Brunet, Stanford University, UNITED STATES

Received: February 17, 2021

Accepted: August 17, 2021

Published: September 10, 2021

Peer Review History: PLOS recognizes the benefits of transparency in the peer review process; therefore, we enable the publication of all of the content of peer review and author responses alongside final, published articles. The editorial history of this article is available here: https://doi.org/10.1371/journal.pgen.1009432

Copyright: $\odot 2021 \mathrm{Li}$ et al. This is an open access article distributed under the terms of the Creative Commons Attribution License, which permits unrestricted use, distribution, and reproduction in any medium, provided the original author and source are credited.

Data Availability Statement: All ChIP-seq data are available in the NCBI BioProject repository (accession number PRJNA699886). (https://www.

\section{Abstract}

Epigenetic alterations occur as organisms age, and lead to chromatin deterioration, loss of transcriptional silencing and genomic instability. Dysregulation of the epigenome has been associated with increased susceptibility to age-related disorders. In this study, we aimed to characterize the age-dependent changes of the epigenome and, in turn, to understand epigenetic processes that drive aging phenotypes. We focused on the aging-associated changes in the repressive histone marks $\mathrm{H} 3 \mathrm{~K} 9 \mathrm{me} 3$ and $\mathrm{H} 3 \mathrm{~K} 27 \mathrm{me} 3$ in C. elegans. We observed region-specific gain and loss of both histone marks, but the changes are more evident for H3K9me3. We further found alteration of heterochromatic boundaries in aged somatic tissues. Interestingly, we discovered that the most statistically significant changes reflected H3K9me3-marked regions that are formed during aging, and are absent in developing worms, which we termed "aging-specific repressive regions" (ASRRs). These ASRRs preferentially occur in genic regions that are marked by high levels of H3K9me2 and H3K36me2 in larval stages. Maintenance of high H3K9me2 levels in these regions have been shown to correlate with a longer lifespan. Next, we examined whether the changes in repressive histone marks lead to de-silencing of repetitive DNA elements, as reported for several other organisms. We observed increased expression of active repetitive DNA elements but not global re-activation of silent repeats in old worms, likely due to the distributed nature of repetitive elements in the $C$. elegans genome. Intriguingly, CELE45, a putative short interspersed nuclear element (SINE), was greatly overexpressed at old age and upon heat stress. SINEs have been suggested to regulate transcription in response to various cellular stresses in mammals. It is likely that CELE45 RNAs also play roles in stress response and aging in $C$. elegans. Taken together, our study revealed significant and specific agedependent changes in repressive histone modifications and repetitive elements, providing important insights into aging biology. 
ncbi.nlm.nih.gov/Traces/study/?acc= PRJNA699886).

Funding: This work was supported by R01 grant AG024425 from the National Institute on Aging (https://www.nia.nih.gov/) and the Cornell University Center for Vertebrate Genomics (https:// cvg.cornell.edu) seed grant to SSL. The funders had no role in study design, data collection and analysis, decision to publish, or preparation of the manuscript.

Competing interests: The authors have declared that no competing interests exist.

\section{Author summary}

Heterochromatin refers to the portion of the genome that is tightly packed where genes stay silent. Heterochromatin is typically decorated by particular chemical groups called histone modifications, such as trimethylation of lysine 9 or lysine 27 on histone 3 (H3K9me3 or H3K27me3). To understand how the heterochromatin landscape may change from a "youthful" to an "aged" state, we monitored the genome-wide patterns of $\mathrm{H} 3 \mathrm{~K} 9 \mathrm{me} 3$ and $\mathrm{H} 3 \mathrm{~K} 27 \mathrm{me} 3$ during aging using the genetic model soil worm C. elegans. We found that while $\mathrm{H} 3 \mathrm{~K} 27 \mathrm{me} 3$ remained relatively stable with age, $\mathrm{H} 3 \mathrm{~K} 9 \mathrm{me} 3$ showed substantial gain and loss at specific loci in aged worms. We observed that new H3K9me3marked heterochromatin preferentially formed in specific gene-rich regions in aged worms. Interestingly, these particular regions were marked by high levels of three other histone modifications when worms were young. This result suggested that $\mathrm{H} 3 \mathrm{~K} 9 \mathrm{me} 3$ gain during aging is influenced by the gene-specific landscape of histone modifications established at young age rather than that it occurs in a stochastic manner. In summary, our study discovered reproducible and gene-specific changes in histone modifications that likely contribute to the aging phenotypes.

\section{Introduction}

In eukaryotes, heterochromatin is the condensed portion of the genome that is typically located at the nuclear periphery and is transcriptionally repressed [1]. The association between aging and dysregulated repressive heterochromatin have been observed across species, from yeast to humans [2-8]. In yeast, loss of transcriptional silencing occurs during replicative senescence and contributes to age-related sterility [2-4]. In flies, heterochromatin levels positively correlate with fly lifespan; Breakdown of heterochromatin at old age contributes to derepression of ribosomal RNAs and genome instability [5]. In worms, aging is associated with loss of peripheral heterochromatin and reduced levels of repressive histone modifications $[6,9,10]$. In mice, the expression of repetitive DNA elements progressively increases in senescent cells and aging somatic tissues [7,11]. In humans, loss of heterochromatin-associated markers and increased expression of repetitive DNA elements are hallmarks of aging in cultured cells from normally aged individuals and from patients with Hutchinson-Gilford progeria syndrome (HGPS) [12-14]. Taken together, the deterioration of heterochromatin structure and loss of transcriptional repression are characteristic features of aging.

Heterochromatic regions are typically decorated with one of the two evolutionarily conserved histone modifications, tri-methylation on histone $\mathrm{H} 3$ at lysine 9 (H3K9me3) and trimethylation on histone $\mathrm{H} 3$ at lysine 27 (H3K27me3). In general, H3K9me3 is associated with constitutive heterochromatin, which covers repeat-rich, gene-poor regions near centromeres and telomeres [15]. H3K9me3 is implicated in suppressing endogenous retrovirus and repetitive DNA elements [16,17]. H3K27me3, on the other hand, is localized at facultative heterochromatin, which forms in gene-rich regions and conditionally represses target genes in a cellular context-dependent and DNA sequence-specific manner. H3K27me3 is involved in silencing developmental genes and X-chromosome inactivation [18,19]. Previous studies have reported both aging-associated gain and loss of repressive histone modifications (H3K9me3 and $\mathrm{H} 3 \mathrm{~K} 27 \mathrm{me} 3$ ). For instance, a global decrease in $\mathrm{H} 3 \mathrm{~K} 9 \mathrm{me} 3$ and $\mathrm{H} 3 \mathrm{~K} 27 \mathrm{me} 3$ levels were observed during aging in whole worms and human cells [9,10,13,20,21]. In flies, the levels of $\mathrm{H} 3 \mathrm{~K} 9 \mathrm{me} 3$ were found to decrease in the aging intestine [22] but to increase in the aging brain [23]. In mice, the levels of $\mathrm{H} 3 \mathrm{~K} 27 \mathrm{me} 3$ reduced in senescent fibroblast cells [20] but elevated in 
the brain of a mouse model of accelerated aging [24]. Therefore, it remains unclear how ageassociated changes in $\mathrm{H} 3 \mathrm{~K} 9 \mathrm{me} 3$ and $\mathrm{H} 3 \mathrm{~K} 27$ me 3 levels contribute to heterochromatin deterioration and the aging phenotypes. It has been proposed that it is not the net abundance but rather the genomic distribution and utilization of the histone modifications that regulate the aging process [25].

Caenorhabditis elegans lacks cytologically dense-staining heterochromatin due to its holocentric chromosomes with dispersed centromeres. Repressive H3K9me3 and H3K27me3 in worms are predominantly localized on repeat-enriched distal arms of all five autosomes, where their distributions are largely overlapped [26-28]. In contrast, heterochromatin is mostly marked by either $\mathrm{H} 3 \mathrm{~K} 9 \mathrm{me} 3$ or $\mathrm{H} 3 \mathrm{~K} 27 \mathrm{me} 3$ in monocentric species, such as flies and humans [28]. Moreover, H3K27me3-marked regions that lack H3K9me3 can be found in the central regions of autosomes and on the $\mathrm{X}$ chromosome in worms. In this study, we investigated how $\mathrm{H} 3 \mathrm{~K} 9 \mathrm{me} 3$ and $\mathrm{H} 3 \mathrm{~K} 27 \mathrm{me} 3$ change during aging in the somatic tissues of germlineless $g l p-1$ mutants by chromatin immunoprecipitation (ChIP) followed by deep sequencing. We found site-specific loss and gain of $\mathrm{H} 3 \mathrm{~K} 9 \mathrm{me} 3$ and $\mathrm{H} 3 \mathrm{~K} 27 \mathrm{me} 3$ in aged somatic cells. Agedependent loss of repressive marks was predominantly found in the central regions of autosomes while gain was localized on the distal autosomal arms. Furthermore, region-specific gain of $\mathrm{H} 3 \mathrm{~K} 9 \mathrm{me} 3$ at old age resulted in the formation of new H3K9me3-marked heterochromatic regions, which we termed "aging-specific repressive regions" (ASRRs). These ASRRs were preferentially localized at genic regions marked by $\mathrm{H} 3 \mathrm{~K} 9 \mathrm{me} 2$ and $\mathrm{H} 3 \mathrm{~K} 36 \mathrm{me} 1 / 2$ at the juvenile stages. We hypothesized that the formation of ASRRs was a result of $\mathrm{H} 3 \mathrm{~K} 9 \mathrm{me} 2$-to$\mathrm{H} 3 \mathrm{~K} 9 \mathrm{me} 3$ conversion and that the trimethylation of $\mathrm{H} 3 \mathrm{~K} 9 \mathrm{me} 2$ could contribute to structural and functional changes of chromatin at old age. We next examined whether somatic aging in the $g l p-1$ mutants is associated with loss of transcriptional suppression of repetitive DNA elements. Although we did find an increase in the levels of RNAs derived from repetitive DNA elements at old age, that nevertheless represented a small fraction $(<1 \%)$ of the overall transcriptome. Interestingly, we found CELE45, a short interspersed nuclear element (SINE), to be significantly over-represented among the repeats with elevated expression in aged worms. In murine and human cells, SINE RNAs were induced and implicated in cellular stress response [29-31]. To this end, we re-analyzed the publicly available RNA-seq data and revealed that CELE45 was induced upon heat stress in worms. Moreover, our analyses indicated that overexpression of CELE45 in the aged germlineless $g l p-1$ mutants was not due to the culture temperature of $25^{\circ} \mathrm{C}$ and/or lack of germline. These results indicated that CELE45 was specifically induced in response to aging.

\section{Results}

\section{Deterioration of $\mathrm{H} 3 \mathrm{~K} 9 \mathrm{me} 3$ boundaries in aged somatic tissues in C. elegans}

To examine the genome-wide patterns of repressive histone modifications (H3K9me3 and H3K27me3) in young and aged somatic tissues of C. elegans, we performed chromatin-immunoprecipitation followed by sequencing (ChIP-seq) in germlineless worms (S1 Table). We used $g l p-1(e 2141)$ mutants that lack germ cells at the non-permissive temperature $\left(25^{\circ} \mathrm{C}\right)$ to harvest post-mitotic somatic tissues and prepare whole worm extracts [32]. For both repressive histone marks, we had two ChIP-seq biological replicates in each of the two time points (day 2 and day 12 of adulthood). We chose day 2 as the young time point when wild-type worms normally reach peak reproduction, and day 12 as the old time point when $10-20 \%$ of the $g l p-1$ (e2141) population would have died [33]. To compare the similarity of the ChIP-seq replicates, we performed pair-wise Pearson's correlation analysis on H3, H3K9me3, and H3K27me3 ChIP-seq tag coverage in 15kb sliding windows along the genome (S1 Fig). Biological 
replicates for the two repressive marks and $\mathrm{H} 3$ control at the same age had genome-wide correlation coefficients higher than 0.83 , indicating high reproducibility.

Next, we identified H3K9me3- and H3K27me3-enriched peak regions normalized to the control H3, using the broad peak parameters in MACS2 (v2.1.0). We combined all biological replicates for peak calling and merged peaks identified at both time points (day 2 and day 12 adults) in order to generate common regions for downstream age-dependent analyses. We noticed a number of gap regions between two broad peaks showed positive fold enrichment $(\log 2)$ of repressive marks relative to $\mathrm{H} 3$ at both time points (S2A Fig). In these cases, we reasoned that the two neighboring peaks represent one long enrichment domain (S2A Fig). We merged them into a single broad peak and the threshold gap size was empirically determined (S2B and S2C Fig). This last step was intended to more accurately capture the broad nature of the H3K27me3 and H3K9me3 marking. The final peak sets had 12,516 H3K9me3 peak regions (S2 Table), and 5,870 H3K27me3 peak regions (S3 Table). The H3K9me3 peak regions were predominantly enriched in the repeat-rich distal arms of autosomes (S2 Table and S3A Fig). $\mathrm{H} 3 \mathrm{~K} 27 \mathrm{me} 3$ coincided with $\mathrm{H} 3 \mathrm{~K} 9 \mathrm{me} 3$ on the distal autosomal arms but was also enriched on $\mathrm{X}$ chromosome and in the H3K9me3-depleted central regions of autosomes (S3 Table and S3A Fig). The genome-wide distribution of both repressive marks in germlineless $g l p-1$ mutants are similar to those from wild-type L3 larvae as previously published (S3A Fig) [26,27,34]. Indeed, correlation analysis showed significant positive correlations for both H3K9me3 (Pearson's correlation coefficient $=0.52, p$-value $<2.2 \mathrm{e}-16$ ) and $\mathrm{H} 3 \mathrm{~K} 27 \mathrm{me} 3$ peak profiles (Pearson's correlation coefficient $=0.38, p$-value $<2.2 \mathrm{e}-16)$ between our ChIP-seq data in $g l p-1$ adults and published data in wild-type L3 larvae [28,34]. To further evaluate the peak calling results, we compared the distribution of repressive peak regions with our published ChIP-seq data of active histone marks (H3K4me3 and H3K36me3) [33,35]. As expected, the genome-wide distributions of active and repressive marks were largely mutually exclusive (S3B Fig). The mutually exclusive occupancy was most evident between H3K27me3 and H3K36me3 (Pearson's correlation coefficient $=-0.71$ ), similar to earlier observations [36,37].

The $\mathrm{H} 3 \mathrm{~K} 9 \mathrm{me} 3$ and $\mathrm{H} 3 \mathrm{~K} 27 \mathrm{me} 3$ repressive histone marks are generally associated with transcriptional repression. To confirm this, we used our previously published RNA-seq data of day 2 and day $12 g l p-1(e 2141)$ germlineless adults [33] to assess the expression of the genes whose coding sequences overlap with either $\mathrm{H} 3 \mathrm{~K} 9 \mathrm{me} 3$ or $\mathrm{H} 3 \mathrm{~K} 27 \mathrm{me} 3$ peak regions. As expected, the majority of these genes showed no detectable RNA expression at both the young and old time points (64.2\% and $73.6 \%$, respectively). Moreover, genes associated with the repressive peak regions showed significantly lower RNA levels compared to genes in non-peak regions (S4A and S4B Fig). In contrast, the majority of the genes that overlap with our published peak regions of active marks (H3K4me3 and $\mathrm{H} 3 \mathrm{~K} 36 \mathrm{me} 3$ ) in $g l p-1$ adults were actively expressed in at least one time point (69.2\% and $78.2 \%$, respectively) [33,35].

To examine whether there were age-dependent changes in H3K9me3 and H3K27me3 enrichment at ChIP-seq peak regions in germlineless $g l p-1$ animals, we first visualized the similarity between young and old samples by performing multidimensional scaling (MDS) analysis. The MDS plots showed that H3K9me3 and H3K27me3 ChIP-seq experiments were separated and clustered by age (Fig $1 \mathrm{~A}$ and $1 \mathrm{~B}$ ). In comparison, H3 ChIP-seq profiles were highly similar between the two ages (Fig $1 \mathrm{~A}$ and $1 \mathrm{~B}$ ) in germlineless $g l p-1$ animals as previously reported $[33,35]$.

Next, we examined how the average $\mathrm{H} 3 \mathrm{~K} 9 \mathrm{me} 3$ and $\mathrm{H} 3 \mathrm{~K} 27$ me3 signals at ChIP-seq peak regions changed between young and old $g l p-1$ mutants. We included our published H3K4me3 and $\mathrm{H} 3 \mathrm{~K} 36 \mathrm{me} 3 \mathrm{ChIP}$-seq data in the analysis for comparisons [33,35]. At $\mathrm{H} 3 \mathrm{~K} 9 \mathrm{me} 3$ peak regions, we found an overall reduced $\mathrm{H} 3 \mathrm{~K} 9 \mathrm{me} 3$ enrichment at old age, and concomitant changes for the active histone marks $\mathrm{H} 3 \mathrm{~K} 4 \mathrm{me} 3$ and $\mathrm{H} 3 \mathrm{~K} 36 \mathrm{me} 3$ at the boundaries. In young 
A

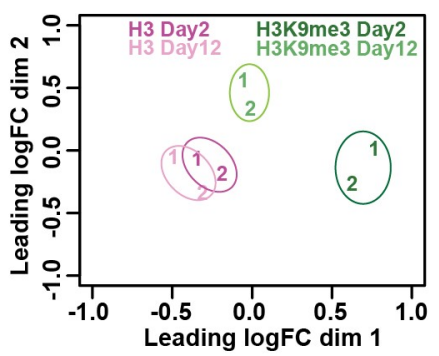

B

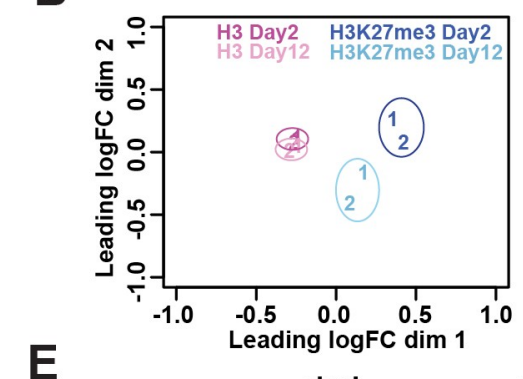

C

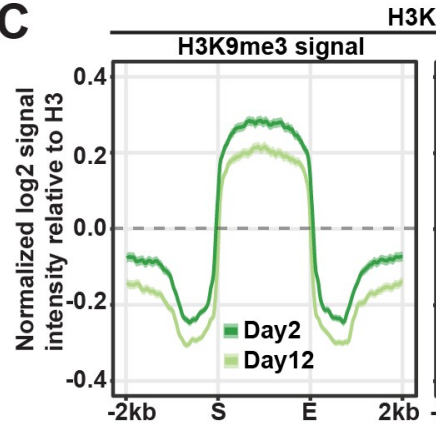

H3K9me3 peak regions $(n=12,516)$
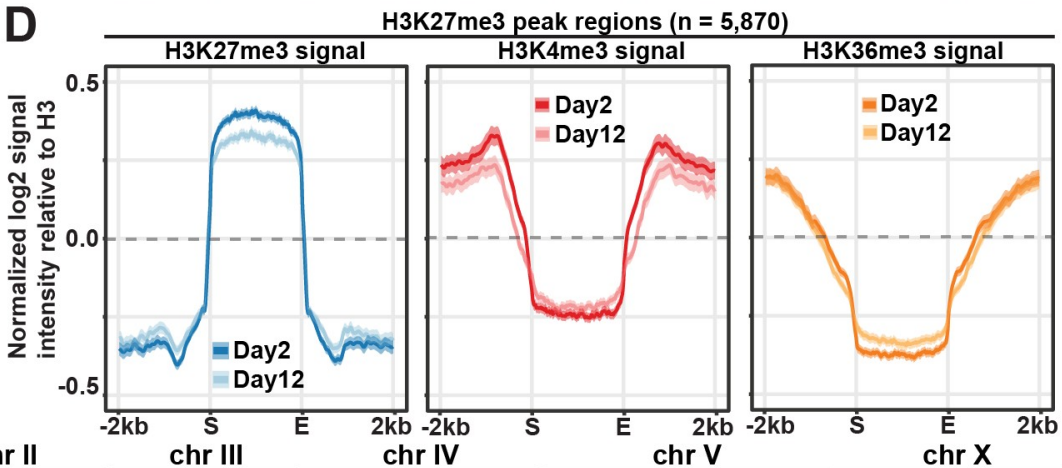

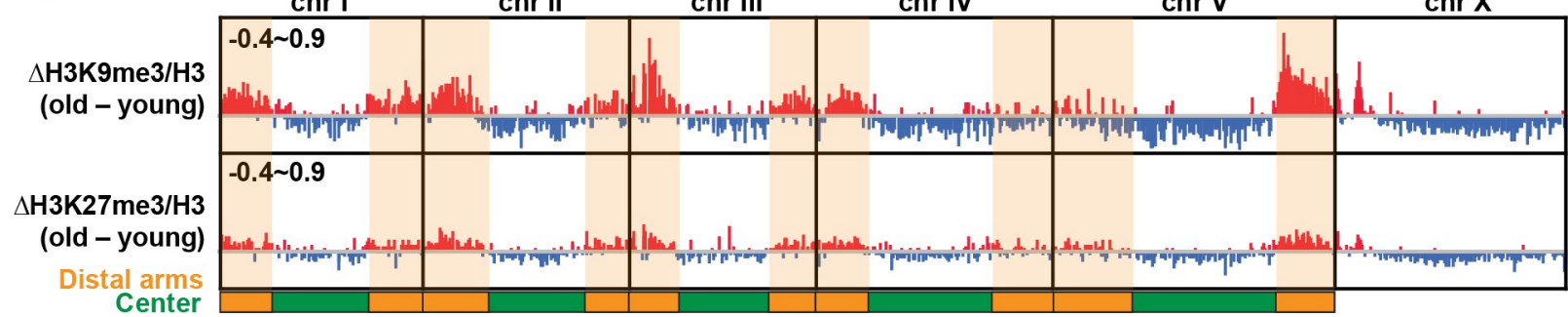

Fig 1. Region-specific gain and loss of repressive marks in aged somatic tissues. Multidimensional scaling (MDS) plots showing the similarity between ChIP-seq replicates of H3 controls (A \& B), H3K9me3 (A), and H3K27me3 (B) in young (day 2 adults) and old (day 12 adults) worms. Two biological replicates are represented by numbers ( 1 and 2). There were age-dependent changes in $\mathrm{H} 3 \mathrm{~K} 9 \mathrm{me} 3$ and $\mathrm{H} 3 \mathrm{~K} 27 \mathrm{me} 3 \mathrm{but}$ not $\mathrm{H} 3$ controls. (C) Metaplots showing the average z-scores of $\mathrm{H} 3 \mathrm{~K} 9 \mathrm{me} 3, \mathrm{H} 3 \mathrm{~K} 4 \mathrm{me} 3$ and $\mathrm{H} 3 \mathrm{~K} 36 \mathrm{me} 3 \mathrm{ChIP}$-seq signal intensity normalized to $\mathrm{H} 3$ (log 2 ) across $\mathrm{H} 3 \mathrm{~K} 9 \mathrm{me} 3$ peak regions $( \pm 2 \mathrm{~kb}$ flanking regions; $\mathrm{n}=12,516)$. The average $\mathrm{H} 3 \mathrm{~K} 9 \mathrm{me} 3$ enrichment across all peaks was reduced with age. The sharp decline of H3K4me3 and H3K36me3 at the boundaries of H3K9me3 peaks was concomitantly lessened with age. (D) Metaplots showing the average $\mathrm{z}$-scores of $\mathrm{H} 3 \mathrm{~K} 27 \mathrm{me} 3, \mathrm{H} 3 \mathrm{~K} 4 \mathrm{me} 3$ and $\mathrm{H} 3 \mathrm{~K} 36 \mathrm{me} 3 \mathrm{ChIP}$-seq signal intensity normalized to H3 (log 2 ) across $\mathrm{H} 3 \mathrm{~K} 27 \mathrm{me} 3$ peak regions ( $\pm 2 \mathrm{~kb}$ flanking regions; $\mathrm{n}=5,870$ ). The average H3K27me3 enrichment across all peaks was reduced with age. (C-D) The solid lines represent the average $\mathrm{z}$-scores with shaded areas showing the $95 \%$ confidence intervals. (E) IGV browser view showing age-dependent changes (old-young) in the average $\mathrm{z}$-scores of normalized H3K9me3 and H3K27me3 signal intensity along the entire genome in 20 -bp bins. Red and blue bars indicate an increase and decrease, respectively, in the average z-scores. The numbers on the top left corner of each track indicate the $y-$ axis range. Distal arms of autosomes are highlighted by the orange shaded regions, and central regions are shaded green.

https://doi.org/10.1371/journal.pgen.1009432.g001

somatic tissues, $\mathrm{H} 3 \mathrm{~K} 9 \mathrm{me} 3$ peak regions coincided with a sharp fall in the levels of these active histone marks (H3K4me3 and H3K36me3), exhibiting clear boundaries (Fig 1C). This distinctive feature became blurred in aged somatic tissues, indicating invasion of active marks in H3K9me3-marked constitutive heterochromatin during aging. At H3K27me3 peak regions, we also observed decreased H3K27me3 enrichment, but the more gradual decline of active histone marks at the boundaries remained stable with age (Fig 1D). It was known that the mutually exclusive patterns of $\mathrm{H} 3 \mathrm{~K} 27 \mathrm{me} 3$ and $\mathrm{H} 3 \mathrm{~K} 36 \mathrm{me} 3$ were maintained by the antagonistic relationship between Polycomb repressive complex 2 (MES-2/3/6) and H3K36 methyltransferase (MES-4). We found that the RNA expression of the four genes remained stable during aging [33], which would be consistent with our observation that the H3K27me3 and H3K36me3 boundaries were preserved in aged $g l p-1$ germlineless mutants. 


\section{Region-specific gain and loss of $\mathrm{H} 3 \mathrm{~K} 9 \mathrm{me} 3$ and $\mathrm{H} 3 \mathrm{~K} 27 \mathrm{me} 3$ in aged somatic tissues in C.elegans}

Like other eukaryotes, the C. elegans genome is organized into transcriptionally active and inactive chromatin compartments in the nucleus [38,39]. We next examined whether spatially partitioned heterochromatic chromosome arms and euchromatic central regions show distinct age-dependent changes in $\mathrm{H} 3 \mathrm{~K} 9 \mathrm{me} 3$ and $\mathrm{H} 3 \mathrm{~K} 27 \mathrm{me} 3$ enrichment in $g l p-1$ animals. Interestingly, age-dependent loss in $\mathrm{H} 3 \mathrm{~K} 9 \mathrm{me} 3$ and $\mathrm{H} 3 \mathrm{~K} 27 \mathrm{me} 3$ mainly occurred in the active central regions of autosomes and most of the $\mathrm{X}$ chromosome (Figs 1E and S5A-S5C). In contrast, age-dependent $\mathrm{H} 3 \mathrm{~K} 9 \mathrm{me} 3$ and $\mathrm{H} 3 \mathrm{~K} 27 \mathrm{me} 3$ gain was mostly observed in the heterochromatic regions, including the distal arms of all five autosomes and the left arm of the $\mathrm{X}$ chromosome (Figs 1E, S5B and S5D). Whereas the H3K9me3 gain in heterochromatic arms was highly visible, and the average signal intensity across all peaks in distal arms indicated statistical significance between young and old animals, that of $\mathrm{H} 3 \mathrm{~K} 27 \mathrm{me} 3$ was relatively modest and insignificant (Figs 1E and S5D). In sum, euchromatic central regions became further depleted of both repressive histone marks while heterochromatic distal arms become significantly more enriched for $\mathrm{H} 3 \mathrm{~K} 9 \mathrm{me} 3$ in aged somatic tissues in $g l p-1$ mutants.

To identify the peak regions with significant changes in $\mathrm{H} 3 \mathrm{~K} 9 \mathrm{me} 3$ and $\mathrm{H} 3 \mathrm{~K} 27 \mathrm{me} 3$ with age in the $g l p-1$ mutants, we performed differential analysis using edgeR [40]. We found that H3K9me3 peak regions had more statistically significant changes with age (578 upregulated and 17 downregulated peaks; $4.7 \%$ of all peaks; S4 Table and S6A Fig) than H3K27me3 peak regions (59 upregulated and 7 downregulated peaks; 1.1\% of all peaks; S5 Table and S6B Fig). We noticed a disproportionately large fraction of the H3K9me3 and H3K27me3 differential peak regions gained repressive marks with age $(578$ of $595 \mathrm{H} 3 \mathrm{~K} 9 \mathrm{me} 3$ differential peaks and 59 of $66 \mathrm{H} 3 \mathrm{~K} 27 \mathrm{me} 3$ differential peaks; S6A and S6B Fig). Most of the peak regions that lost repressive $\mathrm{H} 3 \mathrm{~K} 9 \mathrm{me} 3$ or $\mathrm{H} 3 \mathrm{~K} 27 \mathrm{me} 3$ marks were not statistically significant at FDR cutoff of 0.05 . While $\mathrm{H} 3 \mathrm{~K} 9 \mathrm{me} 3$ peak regions substantially overlapped with $\mathrm{H} 3 \mathrm{~K} 27 \mathrm{me} 3$ peak regions ( $80 \%$ of the area), age-dependent changes in H3K9me3 and H3K27me3 largely occurred in distinct domains. On average, only $6.6 \%$ of upregulated and $12.7 \%$ of downregulated peak regions of both repressive marks overlapped.

Similar to S4 Fig, we next divided the repressive peak regions into three groups based on their age-dependent changes (upregulated, downregulated, no significant change) and associated them with gene expression levels based on our previous RNA-seq data [33]. We found that genes overlapping with the non-significant and upregulated repressive peak regions showed significantly lower RNA expression levels compared to non-peak regions (Fig 2A and 2D) as discussed earlier in S4 Fig. Genes in the upregulated H3K9me3 peak regions had significantly higher RNA levels than genes in non-significant $\mathrm{H} 3 \mathrm{~K} 9$ me 3 peak regions at both young and old time points (KS test $p$-value $<0.0001$; Fig $2 \mathrm{~A}$ and $2 \mathrm{~B}$ ), suggesting the genomic regions that showed age-dependent gain in $\mathrm{H} 3 \mathrm{~K} 9 \mathrm{me} 3$ are likely transcriptionally active. In contrast, RNA levels were lower in genes associated with the upregulated $\mathrm{H} 3 \mathrm{~K} 27 \mathrm{me} 3$ peak regions than non-significant $\mathrm{H} 3 \mathrm{~K} 27 \mathrm{me} 3$ peak regions (KS test $p$-value $=0.013$; Fig $2 \mathrm{C}$ and $2 \mathrm{D}$ ). The analysis for genes associated with the downregulated repressive peaks regions was inconclusive due to the small gene count and lack of statistical power.

Next, we examined whether age-dependent changes in the histone marks correlate with gene expression changes in the $g l p-1$ mutants. As a control, we first analyzed our published H3K4me3 peaks and RNA-seq data in the young and old $g l p-1$ mutants [33]. We examined the genes that were differentially expressed at old age $($ FDR $<0.01)$ and overlapped with significantly upregulated and downregulated $\mathrm{H} 3 \mathrm{~K} 4 \mathrm{me} 3$ peaks. We found a strong positive correlation between RNA expression and H3K4me3 changes (Pearson's correlation coefficient $=0.72$; 

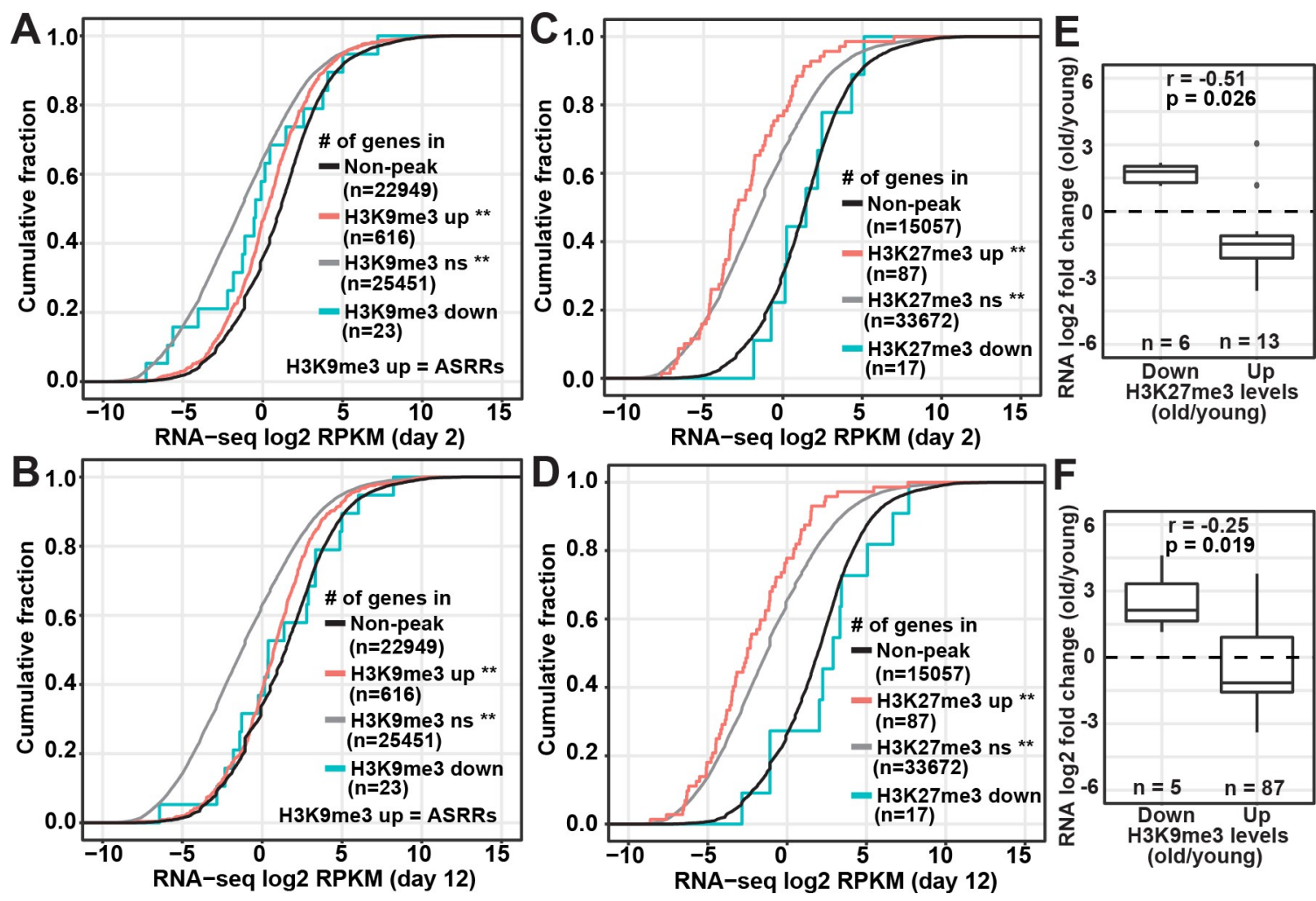

Fig 2. Anti-correlation between changes in repressive marks and gene expression. (A-D) Cumulative frequency plots of the normalized RNA read counts $\left(\log _{2}\right.$ RPKM) of genes associated with H3K9me3 (A and B) or H3K27me3 (C and D) peak regions versus genes in non-peak regions (black curves) in young (day 2) and old (day 12) germlineless $g l p-1$ (e2141) mutants. Repressive peak regions were divided into three groups based on age-dependent changes: significantly upregulated (up, red curves), significantly downregulated (down, cyan curves), and non-significant (ns, gray curves). Comparing to genes in the non-peak regions (black curves), genes associated with upregulated and non-significant repressive peak regions had significantly lower RNA expression levels $\left({ }^{* *}, p\right.$-values of Kolmogorov-Smirnov test $<0.0001)$. The number of genes associated with a given set of peak regions is indicated in the parenthesis. Abbreviation: ASRRs, aging-specific repressive regions. (E and F) Box plots comparing age-dependent RNA expression fold change $\left(\log _{2}\right)$ of differentially expressed genes associated with significantly upregulated or downregulated H3K27me3 (E) or H3K9me3 (F) peaks. The numbers of differentially expressed genes (n) associated with individual groups of peak regions are indicated in the figures: 87 and 5 genes associated with the 578 upregulated and 17 downregulated $\mathrm{H} 3 \mathrm{~K} 9 \mathrm{me} 3$ peaks, and 13 and 6 genes associated with the 59 upregulated and 7 downregulated H3K27me3 peaks, respectively. Pearson's correlation coefficient ( $\mathrm{r}$ ) and $p$-value of the correlation between fold changes in RNA expression and histone modifications are indicated on the top of each panel.

https://doi.org/10.1371/journal.pgen.1009432.g002

S6C Fig), consistent with our previous findings [33]. Next, we examined the differentially expressed genes that overlapped with differential $\mathrm{H} 3 \mathrm{~K} 9 \mathrm{me} 3$ and $\mathrm{H} 3 \mathrm{~K} 27 \mathrm{me} 3$ peak regions. We found a strong negative correlation between changes in $\mathrm{H} 3 \mathrm{~K} 27 \mathrm{me} 3$ and gene expression (Pearson's correlation coefficient $=-0.51$; Fig 2E). The age-dependent changes in H3K9me3 only weakly anti-correlated with gene expression changes (Pearson's correlation coefficient $=$ -0.25 ; Fig 2F). It is however important to note that H3K9me3, and H3K27me3, might influence the expression of genes whose coding sequences do not directly overlap with their peak regions, which would be difficult to assess based on the available data.

\section{New H3K9me3-marked repressive domains were formed in aged somatic tissues}

To gain more insights into the genes associated with differential repressive peak regions, we used WormCat to identify their enriched gene ontology (GO) terms [41] and WormBase enrichment analysis tool to identify their tissue specificity and phenotype clusters [42]. We 

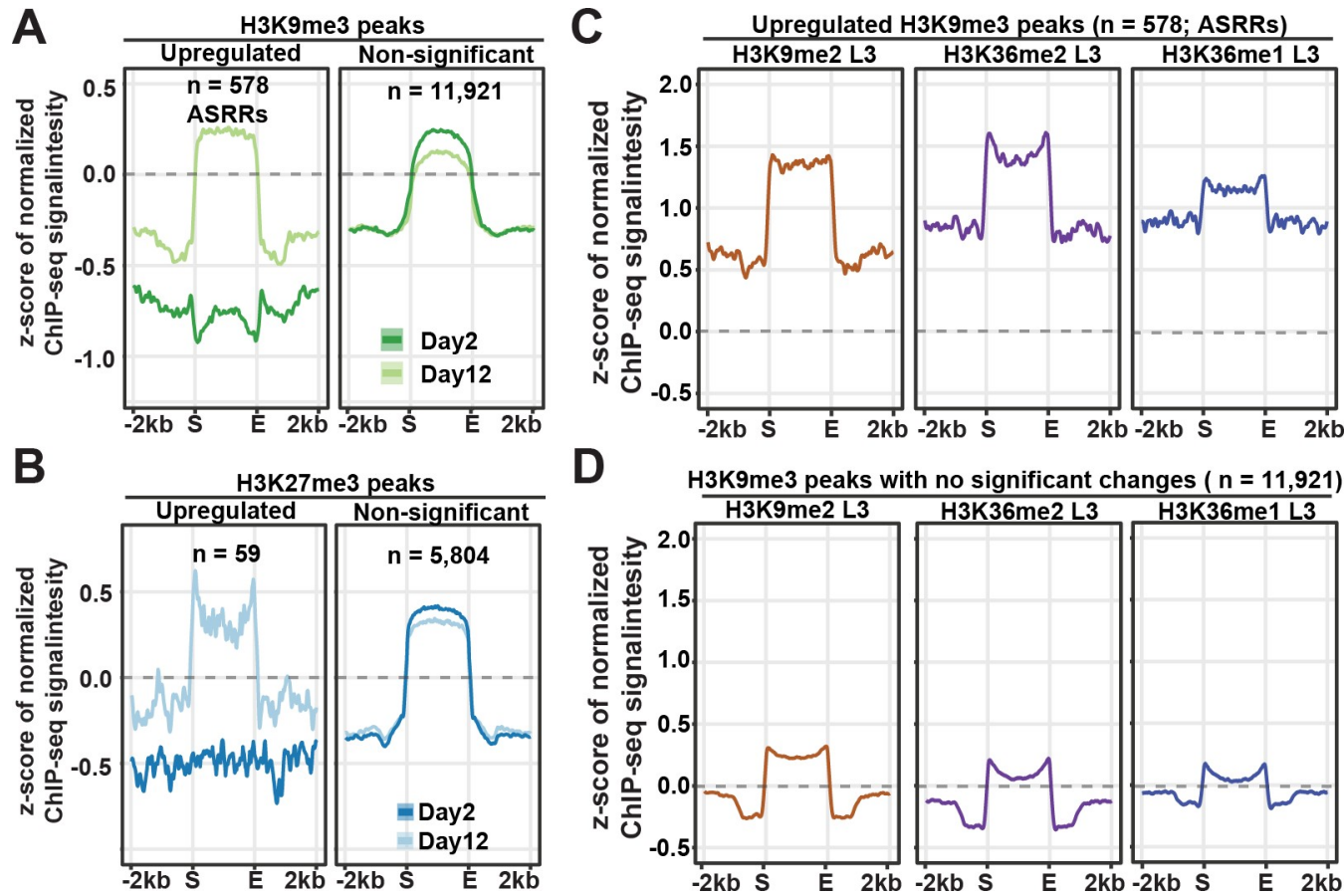

Fig 3. Age-dependent gain of $\mathrm{H} 3 \mathrm{~K} 9 \mathrm{me} 3$ in regions marked by $\mathrm{H} 3 \mathrm{~K} 9 \mathrm{me} 2$ and $\mathrm{H} 3 \mathrm{~K} 36 \mathrm{me} 1 / 2$. (A-B) Metaplots showing the average $\mathrm{z}$-scores of ChIP-seq signal intensity normalized to $\mathrm{H} 3\left(\log _{2}\right)$ in $\mathrm{H} 3 \mathrm{~K} 9 \mathrm{me} 3$ (A) or H3K27me3 (B) peak regions ( $\pm 2 \mathrm{~kb}$ flanking regions) with significant gain or non-significant change with age. (C-D) Metaplots showing the $\mathrm{z}$ scores of $\mathrm{H} 3 \mathrm{~K} 9 \mathrm{me} 2$ and $\mathrm{H} 3 \mathrm{~K} 36 \mathrm{me} 1 / 2$ ChIP-seq signal intensity in peak regions with significant gain $(\mathrm{C})$ or no significant change (D) in H3K9me3 levels during aging. The grey dashed lines indicate the z-score of zero.

https://doi.org/10.1371/journal.pgen.1009432.g003

found no significant enrichment in tissue types and phenotypes for genes associated with downregulated $\mathrm{H} 3 \mathrm{~K} 9 \mathrm{me} 3$ and $\mathrm{H} 3 \mathrm{~K} 27 \mathrm{me} 3$ peak regions. Hereafter, we focused on the genes associated with the upregulated $\mathrm{H} 3 \mathrm{~K} 9 \mathrm{me} 3$ and $\mathrm{H} 3 \mathrm{~K} 27$ me3 peak regions. In the GO enrichment analysis, upregulated $\mathrm{H} 3 \mathrm{~K} 9 \mathrm{me} 3$ peaks were associated with genes overrepresented for the functional terms related to fundamental cellular processes, particularly "chromatin" (S6D Fig). These genes were enriched for cell cycle phenotypes (S6E Fig) and the tissue germline (S6F Fig). Taken together, the GO data suggested that chromatin genes, many with key roles in the cell division cycle and germline development, preferentially accumulate higher levels of H3K9me3 with age.

While most genes associated with repressive H3K9me3 $(n=12,516)$ and H3K27me3 $(n=5,870)$ peak regions were silent, we found that genes associated with significantly upregulated repressive peak regions tends to be actively expressed at both young and old time points in our previously published RNA-seq data $(82.0 \%$ of the genes in the 578 upregulated H3K9me3 peaks and $58.6 \%$ in the 59 upregulated $\mathrm{H} 3 \mathrm{~K} 27 \mathrm{me} 3$ peaks) [33]. One possibility was that the regions that showed statistically significant upregulation of H3K9me3 and H3K27me3 in aged worms had very low levels of the repressive marks in young worms, so the genes in those regions were actively expressed at the young stage. Indeed, the average plots showed that the upregulated repressive peak regions were deprived of $\mathrm{H} 3 \mathrm{~K} 9 \mathrm{me} 3$ and $\mathrm{H} 3 \mathrm{~K} 27 \mathrm{me} 3$ marks at young age (Fig 3A and 3B). At old age, these regions accumulated $\mathrm{H} 3 \mathrm{~K} 9 \mathrm{me} 3$ and $\mathrm{H} 3 \mathrm{~K} 27 \mathrm{me} 3$ at levels comparable to the average of the non-differential peak regions (Fig $3 \mathrm{~A}$ and $3 \mathrm{~B}$ ). This result indicated that certain genomic regions harboring actively expressed genes formed repressive domains by accumulating $\mathrm{H} 3 \mathrm{~K} 9 \mathrm{me} 3$ and $\mathrm{H} 3 \mathrm{~K} 27 \mathrm{me} 3$ in aged somatic tissues in $g l p-$ 
1 mutants. For clarity purposes, the $578 \mathrm{H} 3 \mathrm{~K} 9 \mathrm{me} 3$ peak regions newly formed at old age were hereafter referred to as aging-specific repressive regions (ASRRs).

\section{Age-dependent gain of $\mathrm{H} 3 \mathrm{~K} 9 \mathrm{me} 3$ in genomic domains marked by $\mathrm{H} 3 \mathrm{~K} 9 \mathrm{me} 2$ and $\mathrm{H} 3 \mathrm{~K} 36 \mathrm{mel} / 2$ at young stage}

We discussed earlier a blurring of the boundaries between $\mathrm{H} 3 \mathrm{~K} 9 \mathrm{me} 3$ peak regions and active histone marks (H3K4me3 and H3K36me3) with age (Fig 1C). Next, we examined the levels of active histone marks in upregulated, downregulated, or non-differential $\mathrm{H} 3 \mathrm{~K} 9 \mathrm{me} 3$ peak regions (S7 Fig). In both upregulated (ASRRs) and non-differential H3K9me3 peak regions, we found the blurred boundaries and spreading of $\mathrm{H} 3 \mathrm{~K} 4 \mathrm{me} 3$ into $\mathrm{H} 3 \mathrm{~K} 9 \mathrm{me} 3$ peak regions at old age. Furthermore, unlike the depletion of $\mathrm{H} 3 \mathrm{~K} 36 \mathrm{me} 3$ in non-differential $\mathrm{H} 3 \mathrm{~K} 9 \mathrm{me} 3$ peak regions, upregulated $\mathrm{H} 3 \mathrm{~K} 9 \mathrm{me} 3$ peak regions (ASRRs) exhibited high levels of $\mathrm{H} 3 \mathrm{~K} 36 \mathrm{me} 3$ at both young and old age. This finding suggested the possibility that the differential repressive peak regions are likely associated with distinct histone modifications and protein binding profiles. This prompted us to examine whether age-dependent changes in $\mathrm{H} 3 \mathrm{~K} 9 \mathrm{me} 3$ and H3K27me3 were associated with the presence of other histone marks or binding of specific protein factors. We compared the differential repressive peak regions with the peak profiles of publicly available ChIP-ChIP, ChIP-seq, and ATAC-seq data (S6 Table). We included data for 26 histone marks, 268 transcription/chromatin factors, and one ATAC-seq analysis $[28,33,35,43-48]$. We first used correlation analysis to detect whether two peak profiles were distributed and clustered in similar regions in the genome. We compared the genome-wide distribution of the $\mathrm{H} 3 \mathrm{~K} 9 \mathrm{me} 3$ or $\mathrm{H} 3 \mathrm{~K} 27 \mathrm{me} 3$ peaks that changed significantly with age with the peak profile of each of the 295 available datasets by calculating the peak coverage in $50 \mathrm{~kb}$ sliding windows across the entire genome and performing pair-wise Person's correlation analysis (S8 Fig and S7 Table). Next, we used the permutation test to determine if two sets of peaks have more frequent overlap than expected and computed pair-wise z-scores (S8 Fig and S8 Table). We looked for top-ranked peak profiles in both tests when compared with differential $\mathrm{H} 3 \mathrm{~K} 9 \mathrm{me} 3$ and H3K27me3 peaks during organismal aging. Among all the comparisons, we found that the 578 peak regions with a significant gain in H3K9me3 (ASRRs) strongly correlated with the peak profiles of H3K9me2 and H3K36me1/2 in wild-type L3 larvae and mixedstage populations (Pearson's correlation coefficient $>0.45$ and permutation $z$-score $>34$ ). As expected, two H3K9me2-binding factors (HPL-2, LIN-61) and the H3K9me1/2 methyltransferase (MET-2) also had high correlation scores (Pearson's correlation coefficient $>0.39$ and permutation $z$-score $>23$ ). The remaining pair-wise correlations were relatively weak in at least one of the two analyses and would not be further discussed here. We also did not uncover any strong correlation for the differential $\mathrm{H} 3 \mathrm{~K} 27 \mathrm{me} 3$ peaks. It is important to note that the majority of the publicly available data were from wild-type L3 larvae or mixed-stage embryos. Taken together, the results suggested that significant $\mathrm{H} 3 \mathrm{~K} 9 \mathrm{me} 3$ gain in ASRRs with aging preferentially occurred at genomic regions decorated with $\mathrm{H} 3 \mathrm{~K} 9 \mathrm{me} 2$ and $\mathrm{H} 3 \mathrm{~K} 36 \mathrm{me} 1 / 2$ in juvenile stages.

To better visualize the association of $\mathrm{H} 3 \mathrm{~K} 9 \mathrm{me} 2$ and $\mathrm{H} 3 \mathrm{~K} 36 \mathrm{me} 1 / 2$ with gain in $\mathrm{H} 3 \mathrm{~K} 9 \mathrm{me} 3$ during aging, we ranked all $\mathrm{H} 3 \mathrm{~K} 9 \mathrm{me} 3$ peak regions in descending order according to $\log 2$ fold change (old/young) in $\mathrm{H} 3$-normalized $\mathrm{H} 3 \mathrm{~K} 9 \mathrm{me} 3$, and plotted the signal intensity of the indicated histone marks in heatmap format (S9A Fig). In S9A Fig, we divided H3K9me3 peak regions into three groups based on the fold change (old/young) ( $>1.25$ in group A, 0.75 1.25 in group $\mathrm{B}$ and $<0.75$ in group $\mathrm{C}$ ). We found that the peak regions that gained $\mathrm{H} 3 \mathrm{~K} 9 \mathrm{me} 3$ with age were preferentially associated with higher levels of $\mathrm{H} 3 \mathrm{~K} 9 \mathrm{me} 2$ and $\mathrm{H} 3 \mathrm{~K} 36 \mathrm{me} 1 / 2$ in L3 stage (Group A in S8A Fig). In contrast, H3K9me2 and H3K36me1/2 enrichment were lower or 
depleted in peak regions with stable or decreasing H3K9me3 enrichment with age (Group B and $\mathrm{C}$ in S8A Fig). Metaplots showed the strong enrichment of H3K9me2 and H3K36me1/2 marks in upregulated $\mathrm{H} 3 \mathrm{~K} 9 \mathrm{me} 3$ peak regions (Fig 3C) compared to non-differential peak regions (Fig $3 \mathrm{D}$ ). These results together pointed to increased $\mathrm{H} 3 \mathrm{~K} 9 \mathrm{me} 3$ marking during aging preferentially occurred at regions enriched for $\mathrm{H} 3 \mathrm{~K} 9 \mathrm{me} 2$ and $\mathrm{H} 3 \mathrm{~K} 36 \mathrm{me} 1 / 2$ at the larval stage. It is plausible that the gaining of $\mathrm{H} 3 \mathrm{~K} 9 \mathrm{me} 3$ in the aged $g l p-1$ mutants was a result of agedependent methylation of $\mathrm{H} 3 \mathrm{~K} 9 \mathrm{me} 2$ and conversion into $\mathrm{H} 3 \mathrm{~K} 9 \mathrm{me} 3$ at these specific regions.

Interestingly, Lee et al. [44] recently reported that elevated levels of $\mathrm{H} 3 \mathrm{~K} 9 \mathrm{me} 2$ at specific regions are associated with a longer lifespan. Comparing their H3K9me2 profiles from mixed stage worms revealed a strong statistically significant overlap between the regions that showed an age-dependent gain in $\mathrm{H} 3 \mathrm{~K} 9 \mathrm{me} 3$ in our $g l p-1$ mutant data with the $\mathrm{H} 3 \mathrm{~K} 9 \mathrm{me} 2$ enriched regions in the long-lived strains (S9B Fig). This correlation led us to hypothesize that the agedependent tri-methylation of $\mathrm{H} 3 \mathrm{~K} 9$ at specific genomic regions we observed in the $g l p-1$ mutant is accompanied by concomitant loss of $\mathrm{H} 3 \mathrm{~K} 9 \mathrm{me} 2$ marking, which might contribute to the aging phenotype.

\section{The expression of CELE45 retrotransposon family increased during aging}

$\mathrm{H} 3 \mathrm{~K} 9 \mathrm{me} 2 / 3$, hallmarks of heterochromatin, are enriched in repeat-rich DNA domains to repress the transcription of repeat elements such as transposons and satellites. In C. elegans, genetic mutations compromising $\mathrm{H} 3 \mathrm{~K} 9$ methylation or heterochromatic factors caused derepression of repeat elements $[48,49]$. Although the repeat-rich distal arms of autosomes showed increased $\mathrm{H} 3 \mathrm{~K} 9 \mathrm{me} 3$ enrichment in aged somatic tissues (S5B Fig), a previous study observed a global loss of $\mathrm{H} 3 \mathrm{~K} 9 \mathrm{me} 3$ abundance during aging in $g l p-1$ mutants in Western blotting [9]. Therefore, we examined whether repeat elements could become re-activated and overexpressed in aged somatic tissues. To this end, we analyzed the locus-specific expression of 62,331 repetitive DNA elements based on Dfam 2.0 annotation in the ribo-minus total RNAseq data of day 2 and day 12 glp-1(e2141) adults $[33,50]$. Repetitive sequences in C. elegans are distributed and well-defined due to its holocentric chromosomes. We found 2,867 (4.6\%) annotated repeat elements had detectable RNA expression in the $g l p-1$ mutants at either the young or old time points, indicating the majority of the repeats remained silenced. Furthermore, only 19 of the 2,867 active repeats were silenced at young age, indicating repeat expression at old age was not due to age-dependent derepression. Although the relative expression of repeat elements increased (159\%) with age (Fig 4A and S9 Table), the overall abundance of repeat RNAs remained relatively low (0.3 0.5\% of the transcriptome) in the $g l p-1$ worms (Fig 4A). Using edgeR analysis, we identified $173(0.28 \%)$ and 75 (0.12\%) repeats with significantly elevated and reduced expression, respectively (S10A Fig and S9 Table). Taken together, these results indicated there was no global age-dependent derepression of silenced repeat elements but a mild increase of the repeat RNAs in the transcriptome $(+0.2 \%$ of the transcriptome) in aged germlineless $g l p-1$ mutants.

To exclude the possibility that there was overexpression of uncharacterized repeats missing in the C. elegans reference genome assembly (ce11/WBcel235), we examined whether the proportion of unmapped RNA reads increased during aging. After subtracting the RNA reads that mapped to annotated repeats, bacteria, and spike-in DNAs, the fraction of unmapped reads in RNA-seq remained stable ( $1.75 \%)$ at both time points (S10B Fig), confirming no overexpression of unannotated sequences in aged somatic tissues.

Next, we grouped the differentially expressed repeats by type (DNA transposon, retrotransposon, satellite, or unknown) and found an overrepresentation of RNA retrotransposons among the repeats whose RNA expression increased at old age (120 of 173; Fig 4B). We then 
A

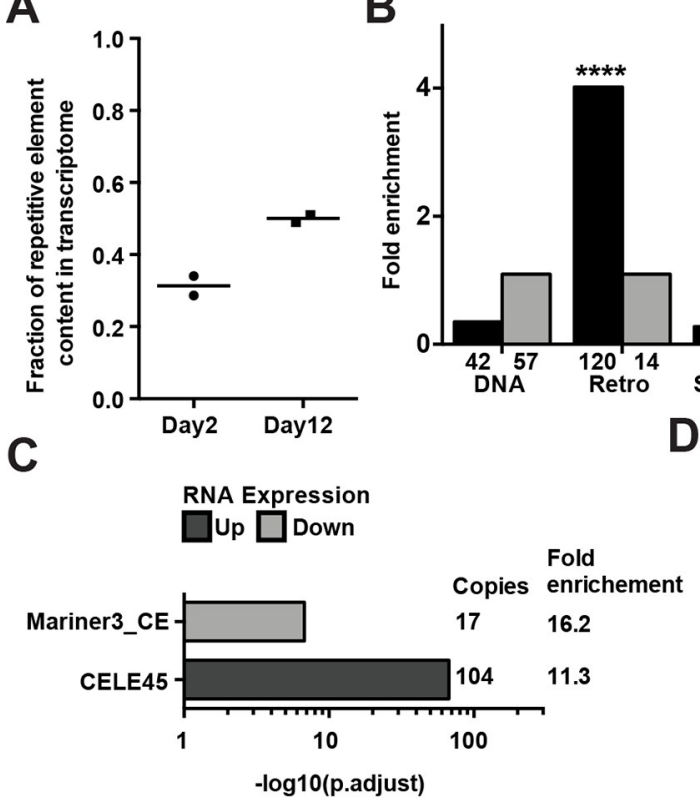

B
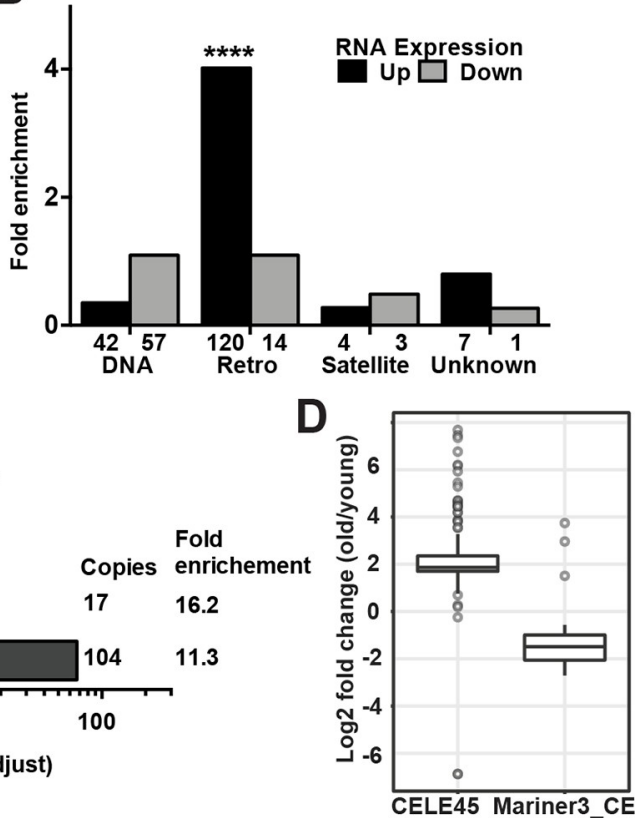

Fig 4. Overexpression of the putative SINE, CELE45, in aged somatic tissues. (A) The percentage of mapped RNA reads that originate from repetitive elements significantly increased during aging (two-tailed t-test, $p$-value $=0.023$ ). Horizontal lines represent the mean of two biological replicates of ribo-depleted RNA-seq in young (day 2) and old (day 12) germline-less $g l p-1(e 2141)$ worms. (B) Bar graphs showing the number of repetitive DNA elements in each group (DNA transposons, retrotransposons, satellite elements and unknown class) (x-axis) that showed significantly increased (Up) or decreased (Down) expression with age. Enrichment analysis revealed a significant overrepresentation of retrotransposon (adjusted $p$-value $=7.14 \times 10^{-68}$ ) among the repeats with significantly elevated expression. (C) Significant enrichment of CELE45 (104 copies) and Mariner3_CE (17 copies) in differentially expressed repeats in aged somatic tissues. (D) The box and whisker plots show RNA fold changes $\left(\log _{2}\right)$ of all expressed copies of CELE45 (150 copies) and Mariner3_CE (40 copies) during aging.

https://doi.org/10.1371/journal.pgen.1009432.g004

grouped the repeats by family and found the DNA transposon family Mariner3_CE and the retrotransposon family CELE45 (Fig 4C) to be overrepresented among the repeats that showed significant age-dependent decrease or increase, respectively, in RNA expression. The Mariner3_CE family represented a tiny fraction of the expressed repeats, and the Mariner3_CE copies appeared to be distributed on all the chromosomes (Figs 4D and S10C). Strikingly, the CELE45 family, which was already well-expressed in young worms, represented $\sim 12.3 \%$ of the expressed REs in the old $g l p-1$ mutants. The CELE45 family is annotated to have 583 copies distributed throughout the genome, with two dense clusters on chromosome X. Our data indicated that most of the actively expressed CELE45 copies (143 of 152) showed a 2-fold or more increase in RNA expression with age, with a somewhat greater induction for the clusters on chr X (Figs 4D and S10D). Since we assigned multi-mapped reads to every matching CELE45 copy, we were concerned about falsely assigning a read from an expressed CELE45 copy to a silent copy that shares high sequence similarity. To address this issue, we performed a similar analysis but only counted uniquely mapped reads, which represented about $31-37 \%$ of the CELE45-mapping reads. This analysis revealed that most of the 56 detectably expressed CELE45 copies showed increased expression during aging ( 23 were statistically significant, with FDR $<0.05$; S10E Fig), supporting that a subset of CELE45 copies distributed across the genome showed age-dependent increased expression in the $g l p-1$ mutants. Since CELE45 is related to the SINE family of retrotransposons, which is known to be transcribed by RNA polymerase III (pol III), we next examined whether the actively expressed CELE45 copies were 
bound by RNA pol III in C. elegans, using the published ChIP-seq data of the RNA polymerase III subunit RPC-1 in wild-type L3 stage (modENCODE 6300). We found most of the active CELE45 copies (95\%) were bound by Pol III, whereas about one-third of the silenced copies (36\%) showed Pol III binding (S11A Fig), suggesting that most of the actively expressed CELE45 copies in $g l p-1$ adults had been actively transcribed starting at larval stages.

To answer whether expression changes of CELE45 and Mariner3_CE copies correlated with age-dependent alterations in repressive marks, we compared the log2 fold changes in RNA and ChIP-seq signals with age. Surprisingly, changes in CELE45 and Mariner3_CE RNA expression did not correlate with changes in $\mathrm{H} 3 \mathrm{~K} 9 \mathrm{me} 3$ and $\mathrm{H} 3 \mathrm{~K} 27 \mathrm{me} 3$ marks during aging (S11B and S11E Fig). Instead, actively expressed CELE45 copies with significantly increased expression at old age were marked with high levels of $\mathrm{H} 3 \mathrm{~K} 9 \mathrm{me} 3$ and H3K27me3 at both young and old age (S11F and S11G Fig). These results indicated that upregulation of CELE45 with aging was not due to derepression by loss of repressive marks.

\section{CELE45 RNA levels increased in response to stress}

We were intrigued by the age-dependent overexpression of the CELE45 in aged somatic tissues in $g l p-1$ mutants. CELE45 is a putative short interspersed nuclear element (SINE). In mammals, SINEs are rapidly induced in response to a variety of cellular stresses, including heat shock and virus infection [29-31]. To this end, we examined whether CELE45 is induced after heat shock in two previously published RNA-seq data in wild-type C. elegans [51,52]. Using the same mapping and differential analyses strategies we applied to our $g l p-1$ aging data, we found that a large number of CELE45 copies showed significantly elevated expression after heat stress in the two independent published data sets [51,52]. In one data set, we found RNA expression of 59 CELE45 copies rapidly increased after short-term heat shock (30 minutes at $33^{\circ} \mathrm{C}$; S9 Table; Brunquell et al. 2016 [51]). In the other data, we found 49 CELE45 copies showed significantly increased expression after 4 hours at $35^{\circ} \mathrm{C}$ (S9 Table; Schreiner et al. 2019 [52]). These results indicated CELE45 was induced in response to heat stress. The age-dependent induction of CELE45 we observed in the $g l p-1$ mutant might result from age-related cellular stress.

In this study, we used germlineless $g l p-1(e 2141)$ worms raised at the non-permissive temperature $\left(25^{\circ} \mathrm{C}\right)$. We asked whether CELE45 induction was a result of lacking germline and/or being cultured at $25 \mathrm{C}$. To this end, we compared the locus-specific expression of repetitive elements between wild-type worms raised at $20^{\circ} \mathrm{C}$ and $g l p-1(e 2141)$ raised at $25^{\circ} \mathrm{C}$ using published RNA-seq data [53]. We found that the expression of repetitive elements in young adults at both conditions was surprisingly similar with very few differentially expressed repeats (12 repeat loci in $\mathrm{S} 9 \mathrm{Table}$ ), indicating that the lack of germline or a culturing temperature between $20 \mathrm{C}$ and $25 \mathrm{C}$ did not significantly impact repeat expression. This observation supported the notion that CELE45 upregulation was a specific response to aging.

\section{Discussion}

In this study, we had investigated changes of $\mathrm{H} 3 \mathrm{~K} 9 \mathrm{me} 3$ and $\mathrm{H} 3 \mathrm{~K} 27 \mathrm{me} 3$ in aged somatic tissues of C. elegans by ChIP-seq. We found that with aging, the overall H3K9me3 levels increased at heterochromatic regions in the distal arms of chromosomes and decreased in euchromatic central regions of autosomes (Fig 1E). H3K27me3 peak regions showed similar patterns of age-dependent changes but at a lower magnitude (Fig 1E). Our data also revealed that 578 peak regions showed statistically significant age-dependent gain of $\mathrm{H} 3 \mathrm{~K} 9 \mathrm{me} 3$ (S6A Fig), and resided in genomic regions not marked by $\mathrm{H} 3 \mathrm{~K} 9 \mathrm{me} 3$ during the juvenile and young adult stage (Fig $3 \mathrm{~A}$ ), thus representing aging-specific repressive regions (ASRRs). Newly 
formed ASRRs at old age were enriched for $\mathrm{H} 3 \mathrm{~K} 9 \mathrm{me} 3$ mark but not $\mathrm{H} 3 \mathrm{~K} 27 \mathrm{me}$, whereas the majority of heterochromatic regions formed during C. elegans development were dually marked by $\mathrm{H} 3 \mathrm{~K} 9 \mathrm{me} 3$ and $\mathrm{H} 3 \mathrm{~K} 27 \mathrm{me}$. It is important to note that we used whole worm extracts of germlineness $g l p-1$ mutants for this study, and therefore our data could not distinguish whether changes in $\mathrm{H} 3 \mathrm{~K} 9 \mathrm{me} 3$ and $\mathrm{H} 3 \mathrm{~K} 27 \mathrm{me} 3$ occurred in all or a specific subset of aged somatic tissues. Nevertheless, the reproducible and robust gain of H3K9me3 in ASRRs detected by ChIP-seq at old age suggested that it likely occurs in a substantial fraction of the aged somatic tissues. Future studies investigating tissue-specific $\mathrm{H} 3 \mathrm{~K} 9 \mathrm{me} 3$ profiles through a developmental and aging time course will help address this question.

Most healthy eukaryotic cells have either round- or oval-shaped nuclei. Abnormal nuclear morphology, which can induce gene expression changes [54,55], occurs during natural and premature aging in worms, flies, and humans $[6,13,56]$. Many previous studies have linked depletion or defects of lamins, the major structural proteins at the nuclear periphery, to the changes in nuclear morphology [57]. However, a recent study provided evidence that altered repressive histone modifications are sufficient to cause nuclear blebbing without detectable changes in lamins [58]. Therefore, it will be important to investigate whether the region-specific gain and loss of repressive marks observed in this study are linked to nuclear abnormalities during aging in future studies.

Our analysis revealed that ASRRs occur in genomic regions marked by high levels of $\mathrm{H} 3 \mathrm{~K} 9 \mathrm{me} 2$ and $\mathrm{H} 3 \mathrm{~K} 36 \mathrm{me} 2$ at juvenile stages. $\mathrm{H} 3 \mathrm{~K} 9 \mathrm{me} 2$ is a histone mark co-localized with heterochromatin tethered to the nuclear lamina [59], whereas $\mathrm{H} 3 \mathrm{~K} 36 \mathrm{me} 2$ is an active histone mark associated with actively transcribed genes [60]. H3K9me2-marked heterochromatin has been reported to be transcriptionally permissive in yeast, worms, and flies [61-64]. Here, we found that ASRRs preferentially overlap with gene bodies (92\%, 529 of 578 ASRRs), and the majority of the overlapped genes are transcriptionally active in somatic tissues [33]. Importantly, our data indicated that the $\mathrm{H} 3 \mathrm{~K} 9 \mathrm{me} 3$ gain in ASRRs at old age is associated with transcriptional repression (Fig 2B and 2F). Taken together, the reproducible aging-associated H3K9me3 gain and transcriptional suppression in ASRRs may represent biomarkers of somatic aging, which warrant further investigation.

Although $\mathrm{H} 3 \mathrm{~K} 9 \mathrm{me} 2$ and $\mathrm{H} 3 \mathrm{~K} 9 \mathrm{me} 3$ are both regarded as repressive histone marks, recent studies using highly specific antibodies found that they are enriched in different genomic regions and exhibit differential patterns of subnuclear localization [59,65]. H3K9me2 is the evolutionarily conserved mark that tethers heterochromatin to the nuclear lamina at the nuclear periphery. $\mathrm{H} 3 \mathrm{~K} 9 \mathrm{me} 2$ is enriched in peripheral heterochromatin which moves toward the nuclear interior as its $\mathrm{H} 3 \mathrm{~K} 9 \mathrm{me} 2$ levels reduced [66]. On the contrary, $\mathrm{H} 3 \mathrm{~K} 9 \mathrm{me} 3$-marked chromatin is distributed from the nuclear periphery to the interior and not enriched in peripheral heterochromatin that directly interacts with peripheral lamina. Therefore, $\mathrm{H} 3 \mathrm{~K} 9 \mathrm{me} 3$ gain in H3K9me2-marked ASRRs likely reflects aging-associated changes in subnuclear location and chromatin structure of peripheral heterochromatin. Aging-associated alterations in heterochromatin compartments were also indicated by the spreading of active histone marks from the flanking regions into H3K9me3-marked regions, as seen in Fig 1C. Together, these results suggested dysregulation of heterochromatin organization occurs in aged somatic tissues.

Intriguingly, recent studies implicated that $\mathrm{H} 3 \mathrm{~K} 9 \mathrm{me} 2$ participates in lifespan regulation and is necessary for the lifespan extension induced by specific genetic mutations or stresses in C. elegans [44,67]. Reduced levels of $\mathrm{H} 3 \mathrm{~K} 9 \mathrm{me} 2$ at a subset of $\mathrm{H} 3 \mathrm{~K} 9 \mathrm{me} 2$ domains are associated with a shorter lifespan [44]. Based on our results and previous observations, we propose the possibility that loss of $\mathrm{H} 3 \mathrm{~K} 9 \mathrm{me} 2$ and/or gain of $\mathrm{H} 3 \mathrm{~K} 9 \mathrm{me} 3$ in ASRRs at old age contribute to the aging process by suppressing gene expression locally and/or triggering global chromatin 
remodeling. Future experiments to track the dynamic and abundance of $\mathrm{H} 3 \mathrm{~K} 9 \mathrm{me} 2 / 3$ and their functional consequences with age would provide substantial new insights into aging biology.

Across species from flies to mammals, aging is associated with the derepression of transposable elements, which appears coupled with a loss of heterochromatin [11,68-72]. Our analysis in C. elegans supported that there was an aging-associated increase in the expression of repetitive DNA elements. Nevertheless, almost all repeats that were silent at young age remained silent at old age, indicating no global derepression of repetitive elements in aged somatic tissues in germlineless $g l p-1$ mutants (S10A Fig). In C. elegans, age-dependent overexpression of the retrotransposon $\operatorname{Cerl}$ (gypsy/Ty3 family) has been reported in germ cells at $15^{\circ} \mathrm{C}$ [73]. We found no detectable expression of Cerl in our experimental condition (somatic tissues at $25^{\circ} \mathrm{C}$ ), as also noted in the previous study [73]. Instead, we found a small subset of repeats were overexpressed at old age with significant over-representation of the putative SINE, CELE45 (Fig 4C). CELE45 was actively transcribed from early embryogenesis [74] to adulthood (Figs 4 and S11A) in C. elegans. The aging-associated over-expression of CELE45 was not due to loss of repressive histone marks (S11G Fig) nor a response to the lack of germline in glp-1(e2141ts) mutants grown at the non-permissive temperature ( $\mathrm{S} 9 \mathrm{Table}$ ). In humans and mice, the expression of SINE RNAs is elevated by cellular stresses and implicated in regulating the expression of stressresponsive genes [29-31,75-77]. Although it is still unclear whether CELE45 participates in stress responses, we found that CELE45 was induced after heat shock in the published RNA-seq data [51-53]. Therefore, CELE45 over-expression at old age is likely a regulated process in response to aging-associated cellular stresses. Going forward, it will be very interesting to determine whether CELE45 is induced by other types of stresses (e.g., bacterial infection), whether it regulates the expression of stress-associated genes like mammalian SINEs do, and whether the overexpression of CELE45 has a functional role in the aging process. Together, this will help us to understand the possible impact of CELE45 overexpression in somatic aging.

\section{Methods}

\section{C. elegans strains and growth}

$g l p-1(e 2141)$ animals were maintained and propagated at $16^{\circ} \mathrm{C}$. To prepare age-synchronized germlineless $g l p-1($ e2141) adults for ChIP-seq experiments, embryos were harvested via bleaching and grown at $25^{\circ} \mathrm{C}$ on $15-\mathrm{cm}$ nematode growth medium (NGM) plates seeded with $2 \mathrm{~mL}$ of 30X concentrated overnight culture of $E$. coli strain OP50 containing $50 \mu \mathrm{g} / \mathrm{mL}$ carbenicillin and $15 \mu \mathrm{g} / \mathrm{mL}$ tetracycline. Worms were refed with OP50 at day 4 in adulthood. Adult worms (day 2 and day 12) were washed three times with cold M9 buffer and snap-frozen in liquid nitrogen. Worm pellets were stored at $-80^{\circ} \mathrm{C}$ before chromatin immunoprecipitation (ChIP).

\section{ChIP-seq library preparation}

Frozen worm pellets were ground into a fine powder with a mortar and pestle, followed by cross-linking with $1 \%$ formaldehyde in PBS for 10 mins at room temperature. Samples were centrifuged at $4,000 \mathrm{~g}$ for 5 minutes and washed with M9 three times. Afterward, the crosslinked chromatin was resuspended in FA buffer and sonicated with Bioruptor to generate chromatin fragments with the target DNA length of $200 \mathrm{bp}$. Chromatin extracts were incubated with anti-H3 (abcam, ab1791), anti-H3K9me3 (abcam, ab8898), or anti-H3K27me3 (07-449, Millipore) antibodies overnight at $4^{\circ} \mathrm{C}$. The antibody/chromatin complex was precipitated with Protein A-Agarose beads, washed three times in cold FA buffer, and incubated with proteinase $\mathrm{K}$ at $65^{\circ} \mathrm{C}$ overnight to reverse crosslinking. DNA was purified with QIAquick PCR purification kit and used for Illumina sequencing library preparation. Indexed DNA 
libraries were sequenced on the Illumina HiSeq (single-end 50-bp) or NextSeq500 (single-end 75-bp) platforms.

Two independent ChIP-seq experiments were performed. The first experiment (exp1) generated two biological replicates of $\mathrm{H} 3$ and $\mathrm{H} 3 \mathrm{~K} 27 \mathrm{me} 3 \mathrm{ChIP}$-seq. The second (exp2) generated two biological replicates of $\mathrm{H} 3$ and $\mathrm{H} 3 \mathrm{~K} 9 \mathrm{me} 3 \mathrm{ChIP}$-seq.

\section{ChIP-seq data analysis}

Sequencing reads were preprocessed with a quality filter (fastq_quality_filter -q $20-\mathrm{p} 80$ ), and adapter sequences were trimmed using Trim Galore (v0.6.5). Processed reads were mapped to $C$. elegans reference genome (ce11/WBcel235) using bowtie2 (v 2.3.5.1) with the default settings. Mapped reads were extracted, and true multi-mapped reads were removed with samtools (samtools view -hSb -F 4 -q 2). PCR duplicates were removed using samtools (samtools rmdup). For $\mathrm{H} 3 \mathrm{~K} 9 \mathrm{me} 3$ and $\mathrm{H} 3 \mathrm{~K} 27 \mathrm{me} 3$ peak identification, biological replicates of the same experiments were merged for broad peak calling using MACS2 (v2.1.0.2; callpeak -t H3K9me3orH3K27me3.bam -c H3.bam-broad-broad-cutoff 0.7 -g ce -B -m 2 50-nomodel—extsize 200) with a permissive q-value cutoff as described [48]. We took a union set of ChIP-seq peaks from all experiments. Neighboring broad peaks were further merged if the gap is less than $5 \mathrm{~kb}$ and has an overall positive $\log 2$ signal ratio of treatments (H3K9me3 or H3K27me3) over the controls (H3). To identify peak regions with differential enrichment between young and old animals, raw read counts in ChIP-seq peak regions were analyzed using edgeR in $\mathrm{R}$ environment $(\mathrm{FDR}<0.05)$ with the comparison: (day12.treatment-day12.H3)—(day2.treatment-day2.H3).

To compare the similarity between biological replicates, the number of mapped reads was counted in 15kb sliding windows. Pearson's correlation coefficient was calculated between the coverage tracks of individual replicates. To visualize ChIP-seq enrichment profiles in young and old animals, mapped reads were first converted into bedGraph tracks of the $\log 2$ signal ratio of treatments ( $\mathrm{H} 3 \mathrm{~K} 9 \mathrm{me} 3$ or $\mathrm{H} 3 \mathrm{~K} 27 \mathrm{me} 3$ ) over the controls $(\mathrm{H} 3)$ using deeptools (bamCompare-bamfile1 H3K9me3orH3K27me3.bam-bamfile2 H3.bam-binSize 20-operation $\log 2$-scaleFactorsMethod readCount-smoothLength 60-extendReads 200 -of bedgraph). Next, the $\log 2$ signal ratio in bedGraph tracks was transformed into z-scores. Z-score bedGraph tracks were converted into bigWig tracks using UCSC bedGraphToBigWig program. computeMatrix utility in deeptools was used to calculate a matrix of $z$-scores from the bigWig tracks for generating metaplots and heatmaps.

\section{Correlation analysis of peak profiles}

To compare the similarity between peak profiles, we compared their genome-wide distribution and frequency of overlap. We evaluated the genome-wide distribution by calculating the peak area (bp) in 50kb sliding windows across the genome of individual peak profiles. Then, we computed the pair-wise Pearson's correlation coefficients between peak coverage matrices. To determine whether the pair-wise overlap frequency is significantly higher than expected by chance, we used regione $\mathrm{R}$ in the $\mathrm{R}$ environment to calculate $\mathrm{z}$-score and $p$ value by permutation test [78]. We used the function "overlapPermTest" in regioneR with the following arguments $(A=$ peak $1, B=$ peak2, ntimes $=2000$, alternative $=$ "auto", genome $=$ ce11, non. overlapping $=$ TRUE, mc.set.seed $=$ FALSE $)$.

\section{RNA-seq analysis of repetitive DNA elements}

Expression of repetitive elements was analyzed in our previously published ribo-minus RNAseq of young (day 2) and old (day 12) germlineless glp-1(e2141) mutants [33]. Repetitive element reference sequences were created by using Dfam 2.0 annotation. Adaptor-trimmed reads 
were then mapped to the repetitive element reference sequences with bowtie v1.1.2 (-n 1 -a -best -strata). Read counts were assigned to individual repetitive elements using featureCounts in Subread package in R (Liao 2014). Multi-mapped reads were assigned one read count toward each of the mapped features. Repeat elements that were differentially expressed between young and old animals were analyzed by using DESeq2 in the R environment (Benjamini-Hochberg-adjusted $p$-value $<0.01$ ).

\section{Supporting information}

S1 Fig. Heatmap showing genome-wide correlation of ChIP-seq replicates. Biological replicates of individual ChIP-seq experiments were highly correlated. Pair-wise Pearson's correlation coefficient was calculated using genome-wide tag coverage in $15 \mathrm{~kb}$ sliding windows. There were two independent $\mathrm{H} 3$ control and treatment ChIP-seq experiments, H3K27me3 (A and $\mathrm{C}$ ) and $\mathrm{H} 3 \mathrm{~K} 9 \mathrm{me} 3$ (B and D).

(TIF)

S2 Fig. Merging neighboring peak regions with a gap showing positive $\log 2$ fold enrichment of repressive marks. (A) IGV browser screenshot showing z-scores of $\mathrm{H} 3 \mathrm{~K} 9$ me3 signal intensity normalized to $\mathrm{H} 3$ in day 2 and day 12 adults (top). Neighboring peaks separated by gaps with a positive mean z-score (yellow wedges) were merged together (bottom). The numbers on the top left corner of each track indicate the y-axis range. (B) The number of H3K9me3 (B) or H3K27me3 (C) peak regions after merging neighboring peaks with a varying maximum threshold of gap size.

S3 Fig. Repressive H3K9me3 and H3K27me3 were enriched in distal chromosome arms. (A) $\mathrm{H} 3 \mathrm{~K} 9 \mathrm{me} 3$ and H3K27me3 were enriched in the distal arm regions of chromosomes. IGV browser view showing the z-score of normalized $\mathrm{H} 3 \mathrm{~K} 9 \mathrm{me} 3$ or $\mathrm{H} 3 \mathrm{~K} 27$ me3 signal intensity in 20-bp bins across the entire genome. The top four tracks showed the ChIP-seq signal intensity normalized to $\mathrm{H} 3$ in young (day 2) and old (day 12) $g l p-1(e 2141)$ adults from data sets in this study. The bottom two tracks showed the ChIP-seq signal intensity normalized to input DNA in N2 L3 larvae from data sets generated by the modENCODE (5037 and 5045). Red and blue bars indicate positive and negative, respectively, z-score. The numbers on the top left corner of each track indicate the $\mathrm{y}$-axis range (z-score). Distal arms of autosomes are highlighted by the orange shaded regions. (B) The genome-wide distribution of active (H3K4me3 and H3K36me3) and repressive (H3K9me3 and H3K27me3) histone modifications were often mutually exclusive. Heatmap showing pairwise Pearson's correlation coefficient between the genome-wide distribution of peak regions of active and repressive histone modifications in adult worms (left). IGV browser screenshot showing the z-scores of H3-normalized signal intensity of active and repressive histone marks (right). (TIF)

S4 Fig. Genes associated with repressive peaks were expressed at lower levels. (A-B) Cumulative frequency plots of the normalized RNA read counts $\left(\log _{2}\right.$ RPKM) of genes associated with repressive peak regions (red curves) or non-peak regions (black curves). There were net negative changes in the RNA expression levels of genes in H3K9me3 (A) or H3K27me3 (B) peak regions (KS test: ${ }^{* *}, p$-value $<0.0001$ ).

(TIF)

S5 Fig. Repressive marks showed distinct patterns of age-dependent changes in the central and distal arm regions of autosomes. Metaplots showing the normalized ChIP-seq signal intensity (z-score) of individual histone marks (H3K9me3, H3K27me3, H3K4me3 or 
H3K36me3) in young (day 2) and old (day 12) germlineless $g l p-1$ (e2141) mutants. Peak regions in individual panels are $\mathrm{H} 3 \mathrm{~K} 9 \mathrm{me} 3$ peaks in the central regions of autosomes (A), H3K9me3 peaks in the distal arms of autosomes (B), H3K27me3 peaks in the central regions of autosomes (C), and H3K27me3 peaks in the distal arms of autosomes (D). There was age-dependent loss of repressive marks in the central regions and gain of $\mathrm{H} 3 \mathrm{~K} 9 \mathrm{me} 3$ in the distal arms of autosomes. (TIF)

S6 Fig. Gene ontology (GO) analysis and expression changes of genes in differential H3K9me3 or H3K27me3 peaks. MA-plot showing $\log _{2}$ fold changes of $\mathrm{H} 3 \mathrm{~K} 9 \mathrm{me} 3$ (A) and H3K27me3 (B) ChIP-seq read counts in peak regions with age (old/young) in germlineless glp-1(e2141) worms. Peaks with significant changes are highlighted in red (Sig), and the rest are colored in grey (NS, not significant). (C) Boxplots of the RNA $\log _{2}$ fold change of differentially expressed genes associated with differential $\mathrm{H} 3 \mathrm{~K} 4 \mathrm{me} 3$ peaks. RNA expression changes strongly correlated with H3K4me3 changes (Pearson's correlation coefficient $=0.72)$. (D-F) Enrichment analyses of functional terms (D), phenotypes (E), and tissue types (F) were performed on the genes associated with differential $\mathrm{H} 3 \mathrm{~K} 9 \mathrm{me} 3$ and $\mathrm{H} 3 \mathrm{~K} 27 \mathrm{me} 3$ peak regions. (TIF)

S7 Fig. Age-dependent changes of active marks in repressive $\mathrm{H} 3 \mathrm{~K} 9 \mathrm{me} 3$ peak regions. (A) Metaplots showing the average z-scores of H3K9me3, H3K4me3 and H3K36me3 ChIP-seq signal intensity normalized to $\mathrm{H} 3\left(\log _{2}\right)$ across $\mathrm{H} 3 \mathrm{~K} 9 \mathrm{me} 3$ peak regions $( \pm 2 \mathrm{~kb}$ flanking regions: significantly upregulated $(\mathrm{n}=578)$, significantly downregulated $(\mathrm{n}=17)$ and non-significant $(\mathrm{n}=11,921)$. H3K4me3 marks showed blurred boundaries and spreading into $\mathrm{H} 3 \mathrm{~K} 9$ me3 peak regions at old age. $\mathrm{H} 3 \mathrm{~K} 36 \mathrm{me} 3$ were depleted in non-significant $\mathrm{H} 3 \mathrm{~K} 9$ me 3 peaks but enriched in upregulated $\mathrm{H} 3 \mathrm{~K} 9 \mathrm{me} 3$ peaks (ASRRs).

(TIF)

S8 Fig. Flowchart showing analysis steps to identify peak profiles that correlate with differential H3K9me3 and H3K27me3 peak regions. Differential H3K9me3 and H3K27me3 peaks were first compared with the publicly available datasets for their similarity in genome-wide distribution. Peak coverage in 50kb sliding windows was used to calculate pair-wise Pearson's correlation coefficient. Next, we used the permutation test to compute the expected overlapping frequency between peak profiles. A z-score was given as a measure of the strength of association between peak profiles that deviate from expectation. Based on these analyses, upregulated $\mathrm{H} 3 \mathrm{~K} 9 \mathrm{me} 3$ peak regions were strongly associated with $\mathrm{H} 3 \mathrm{~K} 9 \mathrm{me} 2$ and $\mathrm{H} 3 \mathrm{~K} 36 \mathrm{mel} / 2$ peak regions. (TIF)

S9 Fig. Heatmap showing $\mathrm{H} 3 \mathrm{~K} 9 \mathrm{me} 2$ and $\mathrm{H} 3 \mathrm{~K} 36 \mathrm{me} 1 / 2$ signals in $\mathrm{H} 3 \mathrm{~K} 9 \mathrm{me} 3$ peak regions. (A-B) $\mathrm{H} 3 \mathrm{~K} 9 \mathrm{me} 3$ peaks were ranked by $\log _{2}$ fold change with age in descending order from top to bottom and separated into three groups based on $\log _{2}$ fold changes with age $(\mathrm{A},>1.25 ; \mathrm{B}$, 0.75 1.25; C <0.75). (A) Heatmap showing normalized ChIP-seq signal intensity (z-score) of H3K9me3 (young and old $g l p-1$ mutants), H3K9me2 (N2 L3 larvae), and H3K36me1/2 (N2 L3 larvae) in $\mathrm{H} 3 \mathrm{~K} 9$ me3 peak regions. (B) Normalized H3K9me2 ChIP-seq signal intensity (zscore) in $\mathrm{N} 2$ or $w d r-5$ (ok1417) worms were generated by Lee et al. (10.7554/eLife.48498) and plotted here in heatmap format. ChIP-seq data were collected after thawing and growing worms for $<6$ generations (earlyGen), 8-14 generations (midGen) or 20 generations (lateGen).

S10 Fig. Expression analysis of repeat elements at young and old animals. (A) MA-plot showing $\log _{2}$ fold changes of RNA expression of annotated repeats (dfam2.0) with age (old/ 
young) in $g l p-1(e 2141)$ worms. Repeat elements with significant expression changes are highlighted in red (Sig), and the rest are colored in grey (NS, not significant). (B) The percentage of unmapped reads with unidentified origin did not increase with age. (C) The genomic locations and RNA $\log _{2}$ fold changes with age of individual Mariner3_CE copies with detectable expression. (D and E) The genomic locations and $\log _{2}$ fold changes of RNA expression with age of individual CELE45 copies with detectable expression when multi-mapping RNA reads were included (D) or excluded (E). (F) The genomic locations of silent CELE45 copies. (TIF)

S11 Fig. Changes in RNA expression and repressive marks in CELE45 copies. (A) Heatmap showing the Pol III ChIP-seq signal intensity (z-score) centered on the transcription start site (TSS) of silenced and expressed CELE45 copies. (B-E) Scatter plots showing the $\log _{2}$ fold changes of RNA expression and ChIP-seq signal intensity with age. Changes in repressive marks did not account for the overexpression of CELE45 copies and the downregulation of Mariner3_CE copies. (F-G) Metaplots showing the average z-scores of H3K9me3 and $\mathrm{H} 3 \mathrm{~K} 27 \mathrm{me} 3 \mathrm{ChIP}$-seq signal intensity normalized to $\mathrm{H} 3\left(\log _{2}\right)$ in silenced $(\mathrm{F})$ and expressed (G) CELE45 copies. Expressed CELE45 copies were marked by higher levels of H3K9me3 and $\mathrm{H} 3 \mathrm{~K} 27 \mathrm{me} 3$ in both young and old worms.

S1 Table. Summary statistics of ChIP-seq data. (XLSX)

S2 Table. H3K9me3 peak regions. (XLSX)

S3 Table. H3K27me3 peak regions. (XLSX)

S4 Table. Differential H3K9me3 peak regions.

(XLSX)

S5 Table. Differential H3K27me3 peak regions.

(XLSX)

S6 Table. Summary of publicly available ChIP-ChIP, ChIP-seq, and ATAC-seq data sets. (XLSX)

S7 Table. Pair-wise Pearson's correlation coefficients of genome-wide distribution of peak profiles.

(XLSX)

S8 Table. Pair-wise overlap analysis between peak profiles by permutation test. (XLSX)

S9 Table. Differential expression analysis of repetitive DNA elements. (XLSX)

\section{Acknowledgments}

Many thanks to Dr. Charles Danko (Cornell University) and Dr. Sylvia Fischer (Harvard Medical School) for insightful discussion and expert advice. Dr. Jeff Glaubitz, Dr. Minghui Wang, and Dr. Qi Sun (Cornell Bioinformatics Facility) for advice on data analysis. 


\section{Author Contributions}

Conceptualization: Cheng-Lin Li, Mintie Pu, Wenke Wang, Siu Sylvia Lee.

Data curation: Cheng-Lin Li, Mintie Pu, Wenke Wang.

Formal analysis: Cheng-Lin Li.

Funding acquisition: Siu Sylvia Lee.

Investigation: Cheng-Lin Li, Mintie Pu, Wenke Wang, Amaresh Chaturbedi, Felicity J. Emerson.

Methodology: Mintie Pu, Wenke Wang, Amaresh Chaturbedi, Felicity J. Emerson.

Project administration: Siu Sylvia Lee.

Supervision: Siu Sylvia Lee.

Validation: Cheng-Lin Li, Mintie Pu, Wenke Wang.

Writing - original draft: Cheng-Lin Li, Siu Sylvia Lee.

Writing - review \& editing: Cheng-Lin Li, Mintie Pu, Wenke Wang, Amaresh Chaturbedi, Felicity J. Emerson, Siu Sylvia Lee.

\section{References}

1. van Steensel B, Belmont AS. Lamina-Associated Domains: Links with Chromosome Architecture, Heterochromatin, and Gene Repression. Cell. 2017; 169: 780-791. https://doi.org/10.1016/j.cell.2017.04. 022 PMID: 28525751

2. Wareham KA, Lyon MF, Glenister PH, Williams ED. Age related reactivation of an X-linked gene. Nature. 1987; 327: 725-727. https://doi.org/10.1038/327725a0 PMID: 3600770

3. Smeal T, Claus J, Kennedy B, Cole F, Guarente L. Loss of transcriptional silencing causes sterility in old mother cells of S. cerevisiae. Cell. 1996; 84: 633-642. https://doi.org/10.1016/s0092-8674(00) 81038-7 PMID: 8598049

4. Kim S, Villeponteau B, Jazwinski SM. Effect of replicative age on transcriptional silencing near telomeres in Saccharomyces cerevisiae. Biochem Biophys Res Commun. 1996; 219: 370-376. https://doi. org/10.1006/bbrc.1996.0240 PMID: 8604994

5. Larson K, Yan SJ, Tsurumi A, Liu J, Zhou J, Gaur K, et al. Heterochromatin formation promotes longevity and represses ribosomal RNA synthesis. Kim SK, editor. PLoS Genet. 2012; 8: e1002473. https:// doi.org/10.1371/journal.pgen.1002473 PMID: 22291607

6. Haithcock E, Dayani Y, Neufeld E, Zahand AJ, Feinstein N, Mattout A, et al. Age-related changes of nuclear architecture in Caenorhabditis elegans. Proc Natl Acad Sci U S A. 2005; 102: 16690-16695. https://doi.org/10.1073/pnas.0506955102 PMID: 16269543

7. Gaubatz JW, Cutler RG. Mouse satellite DNA is transcribed in senescent cardiac muscle. J Biol Chem. 1990; 265: 17753-17758. PMID: 1698780

8. Oberdoerffer P, Michan S, McVay M, Mostoslavsky R, Vann J, Park SK, et al. SIRT1 Redistribution on Chromatin Promotes Genomic Stability but Alters Gene Expression during Aging. Cell. 2008; 135: 907918. https://doi.org/10.1016/j.cell.2008.10.025 PMID: 19041753

9. Ni Z, Ebata A, Alipanahiramandi E, Lee SS. Two SET domain containing genes link epigenetic changes and aging in Caenorhabditis elegans. Aging Cell. 2012; 11: 315-325. https://doi.org/10.1111/j.14749726.2011.00785.x PMID: 22212395

10. Maures TJ, Greer EL, Hauswirth AG, Brunet A. The H3K27 demethylase UTX-1 regulates C. elegans lifespan in a germline-independent, insulin-dependent manner. Aging Cell. 2011; 10: 980-990. https:// doi.org/10.1111/j.1474-9726.2011.00738.x PMID: 21834846

11. De Cecco M, Criscione SW, Peterson AL, Neretti N, Sedivy JM, Kreiling JA. Transposable elements become active and mobile in the genomes of aging mammalian somatic tissues. Aging (Albany NY). 2013; 5: 867-883. https://doi.org/10.18632/aging.100621 PMID: 24323947

12. Goldman RD, Shumaker DK, Erdos MR, Eriksson M, Goldman AE, Gordon LB, et al. Accumulation of mutant lamin A progressive changes in nuclear architecture in Hutchinson-Gilford progeria syndrome. 
Proc Natl Acad Sci U S A. 2004; 101: 8963-8968. https://doi.org/10.1073/pnas.0402943101 PMID: 15184648

13. Scaffidi P, Misteli T. Lamin A-dependent nuclear defects in human aging. Science (80-). 2006; 312 : 1059-1063. https://doi.org/10.1126/science.1127168 PMID: 16645051

14. LaRocca TJ, Cavalier AN, Wahl D. Repetitive elements as a transcriptomic marker of aging: Evidence in multiple datasets and models. Aging Cell. 2020; 19: 1-6. https://doi.org/10.1111/acel.13167 PMID: 32500641

15. Bannister AJ, Kouzarides T. Regulation of chromatin by histone modifications. Cell Res. 2011; 21: 381395. https://doi.org/10.1038/cr.2011.22 PMID: 21321607

16. Peters AHFM, O'Carroll D, Scherthan H, Mechtler K, Sauer S, Schöfer C, et al. Loss of the Suv39h Histone Methyltransferases Impairs Mammalian Heterochromatin and Genome Stability. Cell. 2001; 107: 323-337. https://doi.org/10.1016/s0092-8674(01)00542-6 PMID: 11701123

17. Matsui T, Leung D, Miyashita H, Maksakova IA, Miyachi H, Kimura H, et al. Proviral silencing in embryonic stem cells requires the histone methyltransferase ESET. Nature. 2010; 464: 927-931. https://doi. org/10.1038/nature08858 PMID: 20164836

18. Margueron R, Reinberg D. The Polycomb complex PRC2 and its mark in life. Nature. 2011; 469: 343349. https://doi.org/10.1038/nature09784 PMID: 21248841

19. Chen Z, Zhang Y. Maternal H3K27me3-dependent autosomal and X chromosome imprinting. Nat Rev Genet. 2020; 21: 555-571. https://doi.org/10.1038/s41576-020-0245-9 PMID: 32514155

20. Bracken AP, Kleine-Kohlbrecher D, Dietrich N, Pasini D, Gargiulo G, Beekman C, et al. The Polycomb group proteins bind throughout the INK4A-ARF locus and are disassociated in senescent cells. Genes Dev. 2007; 21: 525-530. https://doi.org/10.1101/gad.415507 PMID: 17344414

21. Shumaker DK, Dechat $T$, Kohlmaier A, Adam SA, Bozovsky MR, Erdos MR, et al. Mutant nuclear lamin A leads to progressive alterations of epigenetic control in premature aging. Proc Natl Acad Sci U S A. 2006; 103: 8703-8708. https://doi.org/10.1073/pnas.0602569103 PMID: 16738054

22. Jeon HJ, Kim YS, Kim JG, Heo K, Pyo JH, Yamaguchi M, et al. Effect of heterochromatin stability on intestinal stem cell aging in Drosophila. Mech Ageing Dev. 2018; 173: 50-60. https://doi.org/10.1016/j. mad.2018.04.001 PMID: 29625135

23. Wood JG, Hillenmeyer S, Lawrence C, Chang C, Hosier S, Lightfoot W, et al. Chromatin remodeling in the aging genome of Drosophila. Aging Cell. 2010; 9: 971-978. https://doi.org/10.1111/j.1474-9726. 2010.00624.x PMID: 20961390

24. Wang CM, Tsai SN, Yew TW, Kwan YW, Ngai SM. Identification of histone methylation multiplicities patterns in the brain of senescence-accelerated prone mouse 8. Biogerontology. 2010; 11: 87-102. https://doi.org/10.1007/s10522-009-9231-5 PMID: 19434510

25. Maleszewska M, Mawer JSP, Tessarz P. Histone Modifications in Ageing and Lifespan Regulation. Curr Mol Biol Reports. 2016; 2: 26-35. https://doi.org/10.1007/s40610-016-0031-9

26. Liu T, Rechtsteiner A, Egelhofer TA, Vielle A, Latorre I, Cheung MS, et al. Broad chromosomal domains of histone modification patterns in C. elegans. Genome Res. 2011; 21: 227-236. https://doi.org/10. 1101/gr.115519.110 PMID: 21177964

27. Ahringer J, Gasser SM. Repressive Chromatin in Caenorhabditis elegans: Establishment, Composition, and Function. Genetics. 2018; 208: 491-511. https://doi.org/10.1534/genetics.117.300386 PMID: 29378810

28. Ho JWK, Jung YL, Liu T, Alver BH, Lee S, Ikegami K, et al. Comparative analysis of metazoan chromatin organization. Nature. 2014; 512: 449-52. https://doi.org/10.1038/nature13415 PMID: 25164756

29. Fornace AJ, Mitchell JB. Induction of B2 RNA polymerase III transcription by heat shock: enrichment for heat shock induced sequences in rodent cells by hybridization subtraction. Nucleic Acids Res. 1986; 14: 5793-5811. https://doi.org/10.1093/nar/14.14.5793 PMID: 2426659

30. Jang KL, Latchman DS. HSV infection induces increased transcription of Alu repeated sequences by RNA polymerase III. FEBS Lett. 1989; 258: 255-258. https://doi.org/10.1016/0014-5793(89)81667-9 PMID: 2557237

31. Liu W man, Chu W ming, Choudary P V., Schmid CW. Cell stress and translational inhibitors transiently increase the abundance of mammalian SINE transcripts. Nucleic Acids Res. 1995; 23: 1758-1765. https://doi.org/10.1093/nar/23.10.1758 PMID: 7784180

32. Priess JR, Schnabel H, Schnabel R. The glp-1 locus and cellular interactions in early C. elegans embryos. Cell. 1987; 51: 601-611. https://doi.org/10.1016/0092-8674(87)90129-2 PMID: 3677169

33. Pu M, Wang M, Wang W, Velayudhan SS, Lee SS. Unique patterns of trimethylation of histone $\mathrm{H} 3$ lysine 4 are prone to changes during aging in Caenorhabditis elegans somatic cells. PLoS Genet. 2018; 14: 1-26. https://doi.org/10.1371/journal.pgen.1007466 PMID: 29912876 
34. Celniker SE, Dillon LAL, Gerstein MB, Gunsalus KC, Henikoff S, Karpen GH, et al. Unlocking the secrets of the genome. Nature. 2009; 459: 927-930. https://doi.org/10.1038/459927a PMID: 19536255

35. Pu M, Ni Z, Wang M, Wang X, Wood JG, Helfand SL, et al. Trimethylation of Lys 36 on H3 restricts gene expression change during aging and impacts life span. Genes Dev. 2015; 29: 718-731. https://doi.org/ 10.1101/gad.254144.114 PMID: 25838541

36. Yuan W, Xu M, Huang C, Liu N, Chen S, Zhu B. H3K36 methylation antagonizes PRC2-mediated H3K27 methylation. J Biol Chem. 2011; 286: 7983-7989. https://doi.org/10.1074/jbc.M110.194027 PMID: 21239496

37. Gaydos LJ, Rechtsteiner A, Egelhofer TA, Carroll CR, Strome S. Antagonism between MES-4 and Polycomb Repressive Complex 2 Promotes Appropriate Gene Expression in C. elegans Germ Cells. Cell Rep. 2012; 2: 1169-1177. https://doi.org/10.1016/j.celrep.2012.09.019 PMID: 23103171

38. Cabianca DS, Muñoz-Jiménez C, Kalck V, Gaidatzis D, Padeken J, Seeber A, et al. Active chromatin marks drive spatial sequestration of heterochromatin in C. elegans nuclei. Nature. 2019; 569: 734-739. https://doi.org/10.1038/s41586-019-1243-y PMID: 31118512

39. Bian Q, Anderson EC, Yang Q, Meyer BJ. Histone H3K9 methylation promotes formation of genome compartments in Caenorhabditis elegans via chromosome compaction and perinuclear anchoring. Proc Natl Acad Sci. 2020; 202002068. https://doi.org/10.1073/pnas.2002068117 PMID: 32385148

40. Robinson MD, McCarthy DJ, Smyth GK. edgeR: A Bioconductor package for differential expression analysis of digital gene expression data. Bioinformatics. 2009; 26: 139-140. https://doi.org/10.1093/ bioinformatics/btp616 PMID: 19910308

41. Holdorf AD, Higgins DP, Hart AC, Boag PR, Pazour GJ, Walhout AJM, et al. WormCat: An online tool for annotation and visualization of caenorhabditis elegans genome-scale data. Genetics. 2020; 214 : 279-294. https://doi.org/10.1534/genetics.119.302919 PMID: 31810987

42. Angeles-Albores $\mathrm{D}$, Lee $\mathrm{R}$, Chan $\mathrm{J}$, Sternberg $\mathrm{P}$. Two new functions in the WormBase Enrichment Suite. microPublication Biol. 2018; 2015-2017. https://doi.org/10.17912/W25Q2N PMID: 32550381

43. Davis CA, Hitz BC, Sloan CA, Chan ET, Davidson JM, Gabdank I, et al. The Encyclopedia of DNA elements (ENCODE): Data portal update. Nucleic Acids Res. 2018; 46: D794-D801. https://doi.org/10. 1093/nar/gkx1081 PMID: 29126249

44. Lee TW, David HS, Engstrom AK, Carpenter BS, Katz DJ. Repressive H3K9me2 protects lifespan against the transgenerational burden of COMPASS activity in C. Elegans. Elife. 2019; 8: 1-19. https:// doi.org/10.7554/eLife.48498 PMID: 31815663

45. Kranz AL, Jiao CY, Winterkorn LH, Albritton SE, Kramer M, Ercan S. Genome-wide analysis of condensin binding in Caenorhabditis elegans. Genome Biol. 2013; 14: 1-15. https://doi.org/10.1186/gb-201314-10-r112 PMID: 24125077

46. Kudron MM, Victorsen A, Gevirtzman L, Hillier LW, Fisher WW, Vafeados D, et al. The modern resource: genome-wide binding profiles for hundreds of Drosophila and Caenorhabditis elegans transcription factors. Genetics. 2018; 208: 937-949. https://doi.org/10.1534/genetics.117.300657 PMID: 29284660

47. Jänes J, Dong Y, Schoof M, Serizay J, Appert A, Cerrato C, et al. Chromatin accessibility dynamics across C. elegans development and ageing. Elife. 2018; 7: 1-24. https://doi.org/10.7554/eLife.37344 PMID: 30362940

48. McMurchy AN, Stempor P, Gaarenstroom T, Wysolmerski B, Dong Y, Aussianikava D, et al. A team of heterochromatin factors collaborates with small RNA pathways to combat repetitive elements and germline stress. Elife. 2017; 6: 1-30. https://doi.org/10.7554/eLife.21666 PMID: 28294943

49. Zeller P, Padeken J, Van Schendel R, Kalck V, Tijsterman M, Gasser SM. Histone H3K9 methylation is dispensable for Caenorhabditis elegans development but suppresses RNA:DNA hybrid-associated repeat instability. Nat Genet. 2016; 48: 1385-1395. https://doi.org/10.1038/ng.3672 PMID: 27668659

50. Hubley R, Finn RD, Clements J, Eddy SR, Jones TA, Bao W, et al. The Dfam database of repetitive DNA families. Nucleic Acids Res. 2016; 44: D81-D89. https://doi.org/10.1093/nar/gkv1272 PMID: 26612867

51. Brunquell J, Morris S, Lu Y, Cheng F, Westerheide SD. The genome-wide role of HSF-1 in the regulation of gene expression in Caenorhabditis elegans. BMC Genomics. 2016; 17: 1-18. https://doi.org/10. 1186/s12864-015-2294-6 PMID: 26818753

52. Schreiner WP, Pagliuso DC, Garrigues JM, Chen JS, Aalto AP, Pasquinelli AE. Remodeling of the Caenorhabditis elegans non-coding RNA transcriptome by heat shock. Nucleic Acids Res. 2019; 47: 9829 9841. https://doi.org/10.1093/nar/gkz693 PMID: 31396626

53. Habacher C, Guo Y, Venz R, Kumari P, Neagu A, Gaidatzis D, et al. Ribonuclease-Mediated Control of Body Fat. Dev Cell. 2016; 39: 359-369. https://doi.org/10.1016/j.devcel.2016.09.018 PMID: 27746047 
54. Biedzinski S, Agsu G, Vianay B, Delord M, Blanchoin L, Larghero J, et al. Microtubules control nuclear shape and gene expression during early stages of hematopoietic differentiation. EMBO J. 2020; 39: 115. https://doi.org/10.15252/embj.2019103957 PMID: 33089509

55. Aureille J, Buffière-Ribot V, Harvey BE, Boyault C, Pernet L, Andersen T, et al. Nuclear envelope deformation controls cell cycle progression in response to mechanical force. EMBO Rep. 2019; 20: 1-11. https://doi.org/10.15252/embr.201948084 PMID: 31368207

56. Brandt A, Krohne G, Großhans J. The farnesylated nuclear proteins KUGELKERN and LAMIN B promote aging-like phenotypes in Drosophila flies. Aging Cell. 2008; 7: 541-551. https://doi.org/10.1111/j. 1474-9726.2008.00406.x PMID: 18494863

57. Butin-Israeli V, Adam SA, Goldman AE, Goldman RD. Nuclear lamin functions and disease. Trends Genet. 2012; 28: 464-471. https://doi.org/10.1016/j.tig.2012.06.001 PMID: 22795640

58. Stephens AD, Liu PZ, Banigan EJ, Almassalha LM, Backman V, Adam SA, et al. Chromatin histone modifications and rigidity affect nuclear morphology independent of lamins. Mol Biol Cell. 2018; 29: 220-233. https://doi.org/10.1091/mbc.E17-06-0410 PMID: 29142071

59. Poleshko A, Smith CL, Nguyen SC, Sivaramakrishnan P, Wong KG, Murray JI, et al. H3K9me2 orchestrates inheritance of spatial positioning of peripheral heterochromatin through mitosis. Elife. 2019; 8: 124. https://doi.org/10.7554/eLife.49278 PMID: 31573510

60. Rao B, Shibata Y, Strahl BD, Lieb JD. Dimethylation of Histone H3 at Lysine 36 DemarcatesRegulatory and Nonregulatory ChromatinGenome-Wide. Mol Cell Biol. 2005; 25: 9447-9459. https://doi.org/10. 1128/MCB.25.21.9447-9459.2005 PMID: 16227595

61. Jih G, Iglesias N, Currie MA, Bhanu N V., Paulo JA, Gygi SP, et al. Unique roles for histone H3K9me states in RNAi and heritable silencing of transcription. Nature. 2017; 547: 463-467. https://doi.org/10. 1038/nature23267 PMID: 28682306

62. De Wit E, Greil F, Van Steensel B. High-resolution mapping reveals links of HP1 with active and inactive chromatin components. PLoS Genet. 2007; 3: 0346-0357. https://doi.org/10.1371/journal.pgen. 0030038 PMID: 17335352

63. De Lucia F, Ni JQ, Vaillant C, Sun FL. HP1 modulates the transcription of cell-cycle regulators in Drosophila melanogaster. Nucleic Acids Res. 2005; 33: 2852-2858. https://doi.org/10.1093/nar/gki584 PMID: 15905474

64. Garrigues JM, Sidoli S, Garcia B a, Strome S. Defining heterochromatin in C. elegans through genomewide analysis of the heterochromatin protein 1 homolog HPL-2. Genome Res. 2015; 25: 76-88. https:// doi.org/10.1101/gr.180489.114 PMID: 25467431

65. Bessler JB, Andersen EC, Villeneuve AM. Differential localization and independent acquisition of the $\mathrm{H} 3 \mathrm{~K} 9 \mathrm{me} 2$ and $\mathrm{H} 3 \mathrm{~K} 9 \mathrm{me} 3$ chromatin modifications in the Caenorhabditis elegans adult germ line. PLoS Genet. 2010;6. https://doi.org/10.1371/journal.pgen.1000830 PMID: 20107519

66. Kind J, Pagie L, Ortabozkoyun H, Boyle S, De Vries SS, Janssen H, et al. Single-cell dynamics of genome-nuclear lamina interactions. Cell. 2013; 153: 178-192. https://doi.org/10.1016/j.cell.2013.02. 028 PMID: 23523135

67. Tian Y, Garcia G, Bian Q, Steffen KK, Joe L, Wolff S, et al. Mitochondrial Stress Induces Chromatin Reorganization to Promote Longevity and UPRmt. Cell. 2016; 165: 1197-1208. https://doi.org/10.1016/ j.cell.2016.04.011 PMID: 27133166

68. De Cecco M, Criscione SW, Peckham EJ, Hillenmeyer S, Hamm EA, Manivannan J, et al. Genomes of replicatively senescent cells undergo global epigenetic changes leading to gene silencing and activation of transposable elements. Aging Cell. 2013; 12: 247-256. https://doi.org/10.1111/acel.12047 PMID: 23360310

69. Van Meter M, Kashyap M, Rezazadeh S, Geneva AJ, Morello TD, Seluanov A, et al. SIRT6 represses LINE1 retrotransposons by ribosylating KAP1 but this repression fails with stress and age. Nat Commun. 2014; 5: 5011. https://doi.org/10.1038/ncomms6011 PMID: 25247314

70. Maxwell $\mathrm{PH}$, Burhans WC, Curcio MJ. Retrotransposition is associated with genome instability during chronological aging. Proc Natl Acad Sci. 2011; 108: 20376-20381. https://doi.org/10.1073/pnas. 1100271108 PMID: 22021441

71. Li W, Prazak L, Chatterjee N, Grüninger S, Krug L, Theodorou D, et al. Activation of transposable elements during aging and neuronal decline in Drosophila. Nat Neurosci. 2013; 16: 529-531. https://doi. org/10.1038/nn.3368 PMID: 23563579

72. Chen $\mathrm{H}$, Zheng $\mathrm{X}$, Xiao D, Zheng Y. Age-associated de-repression of retrotransposons in the Drosophila fat body, its potential cause and consequence. Aging Cell. 2016; 15: 542-552. https://doi.org/10. 1111/acel.12465 PMID: 27072046 
73. Dennis S, Sheth U, Feldman JL, English KA, Priess JR. C. elegans Germ Cells Show Temperature and Age-Dependent Expression of Cer1, a Gypsy/Ty3-Related Retrotransposon. Ding S-W, editor. PLoS Pathog. 2012; 8: e1002591. https://doi.org/10.1371/journal.ppat.1002591 PMID: 22479180

74. Ansaloni F, Scarpato M, Di Schiavi E, Gustincich S, Sanges R. Exploratory analysis of transposable elements expression in the C. elegans early embryo. BMC Bioinformatics. 2019; 20: 484. https://doi.org/ 10.1186/s12859-019-3088-7 PMID: 31757208

75. Zovoilis A, Cifuentes-Rojas C, Chu HP, Hernandez AJ, Lee JT. Destabilization of B2 RNA by EZH2 Activates the Stress Response. Cell. 2016; 167: 1788-1802.e13. https://doi.org/10.1016/j.cell.2016.11.041 PMID: 27984727

76. Karijolich J, Zhao Y, Alla R, Glaunsinger B. Genome-wide mapping of infection-induced SINE RNAs reveals a role in selective mRNA export. Nucleic Acids Res. 2017; 45: 6194-6208. https://doi.org/10. 1093/nar/gkx180 PMID: 28334904

77. Tucker JM, Glaunsinger BA. Host Noncoding Retrotransposons Induced by DNA Viruses: a SINE of Infection? J Virol. 2017; 91: 1-6. https://doi.org/10.1128/JVI.00982-17 PMID: 28931686

78. Gel B, Díez-Villanueva A, Serra E, Buschbeck M, Peinado MA, Malinverni R. RegioneR: An R/Bioconductor package for the association analysis of genomic regions based on permutation tests. Bioinformatics. 2016; 32: 289-291. https://doi.org/10.1093/bioinformatics/btv562 PMID: 26424858 